Louisiana State University

LSU Digital Commons

$5-28-2010$

\title{
First measurement of the muon neutrino charged current quasielastic double differential cross section
}

\author{
A. A. Aguilar-Arevalo \\ Universidad Nacional Autónoma de México \\ C. E. Anderson \\ Yale University \\ A. O. Bazarko \\ Princeton University \\ S. J. Brice \\ Fermi National Accelerator Laboratory \\ B. C. Brown \\ Fermi National Accelerator Laboratory
}

See next page for additional authors

Follow this and additional works at: https://digitalcommons.Isu.edu/physics_astronomy_pubs

\section{Recommended Citation}

Aguilar-Arevalo, A., Anderson, C., Bazarko, A., Brice, S., Brown, B., Bugel, L., Cao, J., Coney, L., Conrad, J., Cox, D., Curioni, A., Djurcic, Z., Finley, D., Fleming, B., Ford, R., Garcia, F., Garvey, G., Grange, J., Green, C., Green, J., Hart, T., Hawker, E., Imlay, R., Johnson, R., Karagiorgi, G., Kasper, P., Katori, T., Kobilarcik, T., Kourbanis, I., Koutsoliotas, S., Laird, E., Linden, S., \& Link, J. (2010). First measurement of the muon neutrino charged current quasielastic double differential cross section. Physical Review D - Particles, Fields, Gravitation and Cosmology, 81 (9) https://doi.org/10.1103/PhysRevD.81.092005

This Article is brought to you for free and open access by the Department of Physics \& Astronomy at LSU Digital Commons. It has been accepted for inclusion in Faculty Publications by an authorized administrator of LSU Digital Commons. For more information, please contact ir@lsu.edu. 


\section{Authors}

A. A. Aguilar-Arevalo, C. E. Anderson, A. O. Bazarko, S. J. Brice, B. C. Brown, L. Bugel, J. Cao, L. Coney, J. M. Conrad, D. C. Cox, A. Curioni, Z. Djurcic, D. A. Finley, B. T. Fleming, R. Ford, F. G. Garcia, G. T. Garvey, J. Grange, C. Green, J. A. Green, T. L. Hart, E. Hawker, R. Imlay, R. A. Johnson, G. Karagiorgi, P. Kasper, T.

Katori, T. Kobilarcik, I. Kourbanis, S. Koutsoliotas, E. M. Laird, S. K. Linden, and J. M. Link 


\section{MIT}

\section{First measurement of the muon neutrino charged current quasielastic double differential cross section}

The MIT Faculty has made this article openly available. Please share how this access benefits you. Your story matters.

\begin{tabular}{|l|l|}
\hline Citation & $\begin{array}{l}\text { MiniBooNE Collaboration et al. "First measurement of the muon } \\
\text { neutrino charged current quasielastic double differential cross } \\
\text { section." Physical Review D } 81.9 \text { (2010): 092005. @ } 2010 \text { The } \\
\text { American Physical Society. }\end{array}$ \\
\hline As Published & http://dx.doi.org/10.1103/PhysRevD.81.092005 \\
\hline Publisher & American Physical Society \\
\hline \hline Version & Final published version \\
\hline Citable link & http://hdl.handle.net/1721.1/57478 \\
\hline Terms of Use & $\begin{array}{l}\text { Article is made available in accordance with the publisher's } \\
\text { policy and may be subject to US copyright law. Please refer to the } \\
\text { publisher's site for terms of use. }\end{array}$ \\
\hline
\end{tabular}




\section{First measurement of the muon neutrino charged current quasielastic double differential cross section}

A. A. Aguilar-Arevalo, ${ }^{13}$ C. E. Anderson, ${ }^{18}$ A. O. Bazarko, ${ }^{15}$ S. J. Brice, ${ }^{7}$ B. C. Brown, ${ }^{7}$ L. Bugel,${ }^{5}$ J. Cao,${ }^{14}$ L. Coney, ${ }^{5}$ J. M. Conrad, ${ }^{12}$ D. C. Cox,${ }^{9}$ A. Curioni, ${ }^{18}$ Z. Djurcic,${ }^{5}$ D. A. Finley,${ }^{7}$ B. T. Fleming, ${ }^{18}$ R. Ford, ${ }^{7}$ F. G. Garcia, ${ }^{7}$ G. T. Garvey, ${ }^{10}$ J. Grange, ${ }^{8}$ C. Green, ${ }^{7,10}$ J. A. Green,,${ }^{9,10}$ T. L. Hart ${ }^{4}$ E. Hawker, ${ }^{3,10}$ R. Imlay, ${ }^{11}$ R. A. Johnson, ${ }^{3}$ G. Karagiorgi, ${ }^{12}$ P. Kasper, ${ }^{7}$ T. Katori, ${ }^{9,12}$ T. Kobilarcik, ${ }^{7}$ I. Kourbanis, ${ }^{7}$ S. Koutsoliotas, ${ }^{2}$ E. M. Laird,${ }^{15}$ S. K. Linden, ${ }^{18}$ J. M. Link, ${ }^{17}$ Y. Liu, ${ }^{14}$ Y. Liu, ${ }^{1}$ W. C. Louis,${ }^{10}$ K. B. M. Mahn,,${ }^{5}$ W. Marsh,${ }^{7}$ C. Mauger,${ }^{10}$ V. T. McGary, ${ }^{12}$ G. McGregor, ${ }^{10}$ W. Metcalf, ${ }^{11}$ P. D. Meyers,${ }^{15}$ F. Mills, ${ }^{7}$ G. B. Mills, ${ }^{10}$ J. Monroe, ${ }^{5}$ C. D. Moore,${ }^{7}$ J. Mousseau, ${ }^{8}$ R. H. Nelson, ${ }^{4}$

P. Nienaber, ${ }^{16}$ J. A. Nowak, ${ }^{11}$ B. Osmanov, ${ }^{8}$ S. Ouedraogo, ${ }^{11}$ R. B. Patterson, ${ }^{15}$ Z. Pavlovic, ${ }^{10}$ D. Perevalov, ${ }^{1}$ C. C. Polly, ${ }^{7}$ E. Prebys, ${ }^{7}$ J. L. Raaf, ${ }^{3}$ H. Ray, ${ }^{8,10}$ B. P. Roe, ${ }^{14}$ A. D. Russell, ${ }^{7}$ V. Sandberg,,${ }^{10}$ R. Schirato, ${ }^{10}$ D. Schmitz, ${ }^{5}$ M. H. Shaevitz, ${ }^{5}$ F. C. Shoemaker, ${ }^{15, *}$ D. Smith, ${ }^{6}$ M. Soderberg, ${ }^{18}$ M. Sorel,${ }^{5, \dagger}$ P. Spentzouris, ${ }^{7}$ J. Spitz, ${ }^{18}$ I. Stancu, ${ }^{1}$ R. J. Stefanski, ${ }^{7}$ M. Sung, ${ }^{11}$ H. A. Tanaka, ${ }^{15}$ R. Tayloe,${ }^{9}$ M. Tzanov, ${ }^{4}$ R. G. Van de Water, ${ }^{10}$ M. O. Wascko, ${ }^{11,}$ D. H. White, ${ }^{10}$ M. J. Wilking, ${ }^{4}$ H. J. Yang, ${ }^{14}$ G. P. Zeller, ${ }^{7}$ and E. D. Zimmerman ${ }^{4}$

(MiniBooNE Collaboration)

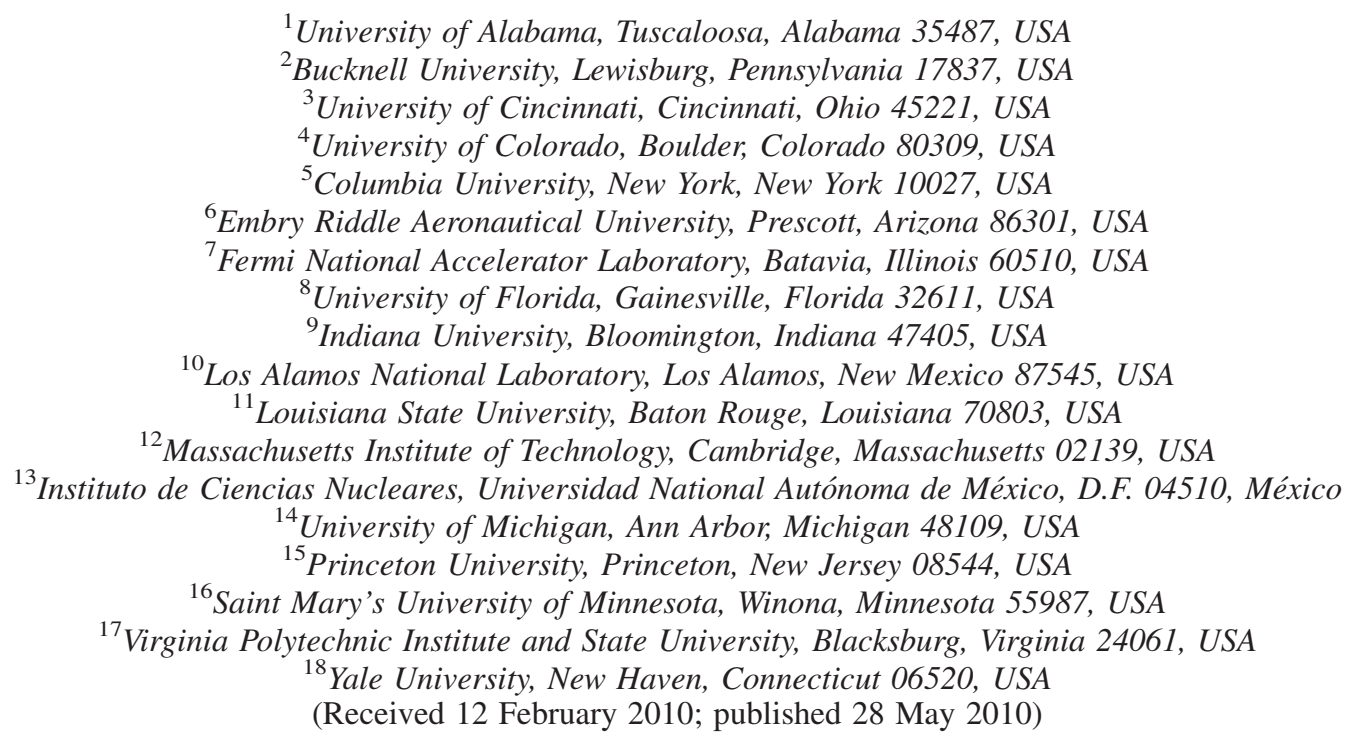

A high-statistics sample of charged-current muon neutrino scattering events collected with the MiniBooNE experiment is analyzed to extract the first measurement of the double differential cross section $\left(\frac{d^{2} \sigma}{d T_{\mu} d \cos \theta_{\mu}}\right)$ for charged-current quasielastic (CCQE) scattering on carbon. This result features minimal model dependence and provides the most complete information on this process to date. With the assumption of CCQE scattering, the absolute cross section as a function of neutrino energy $\left(\sigma\left[E_{\nu}\right]\right)$ and the single differential cross section $\left(\frac{d \sigma}{d Q^{2}}\right)$ are extracted to facilitate comparison with previous measurements. These quantities may be used to characterize an effective axial-vector form factor of the nucleon and to improve the modeling of low-energy neutrino interactions on nuclear targets. The results are relevant for experiments searching for neutrino oscillations.

DOI: $10.1103 /$ PhysRevD.81.092005

\footnotetext{
*Deceased.

${ }^{\dagger}$ Present address: IFIC, Universidad de Valencia and CSIC, Valencia 46071, Spain.

${ }^{*}$ Present address: Imperial College; London SW7 2AZ, United Kingdom.
}

PACS numbers: 25.30.Pt, 13.15.+g, 14.60.Lm, 14.60.Pq

\section{INTRODUCTION}

Neutrino charged-current (CC) scattering without pions in the final state is important to measure and characterize, and is a critical component in the neutrino oscillation program of the MiniBooNE experiment [1-4] at Fermilab. Most of these events are charged-current quasielastic scattering (CCQE) of the muon neutrino on a bound 
nucleon $\left(\nu_{\mu}+n \rightarrow \mu^{-}+p\right)$. A robust model of these interactions is required to support future experiments such as NOvA [5] and T2K [6] that are also searching for $\nu_{\mu} \rightarrow \nu_{e}$ oscillations. Such experiments will use $\nu_{e}$ CC interactions to detect the appearance of any $\nu_{e}$ resulting from oscillations in the large distance between production and detection. Additional use will be made of $\nu_{\mu} \mathrm{CC}$ interactions to normalize the neutrino content at production using a near detector and to search for the disappearance of $\nu_{\mu}$ via a far detector. These analyses will require all available experimental and theoretical insight on the CCQE interaction in the $\approx 1 \mathrm{GeV}$ energy range and on nuclear (carbon, oxygen) targets. While many unknown quantities are eliminated in these experiments by considering ratios of far to near events, the cancellation is not complete due to differences in neutrino flux and backgrounds in the near and far detectors. Thus, in order to permit precision oscillation measurements, it is important to have an accurate characterization of the CCQE differential cross sections over a wide span of neutrino energies.

Historically, it has proven difficult to accurately define the CCQE cross section and precise measurements have been unavailable. The experimental execution and data interpretation are nontrivial for several reasons. Neutrino beams typically span a wide energy range thereby preventing an incoming energy constraint on the reaction. The neutrino flux itself is often poorly known, hampering normalization of reaction rates. Background processes are frequently significant and difficult or impossible to separate from the $\mathrm{CCQE}$ signal, for instance, $\mathrm{CC}$ pion production combined with pion absorption in the nucleus. Further complicating the description, the target nucleon is not free but bound in a nuclear target and correlations between nucleons may be important. There are differing detection strategies employed by different experiments, for example, some require detection of the final-state nucleon and some do not. Finally, the nuclear target often differs between experiments, thus making comparisons less straightforward.

The current data on CCQE scattering come from a variety of experiments operating at differing energies and with different nuclei [7]. Modeling of this data has been consistent from experiment to experiment, yet remains fairly unsophisticated. Preferred for its simplicity, neutrino CCQE models typically employ a relativistic Fermi gas (RFG) model (such as that of Ref. [8]) that combines the bare nucleon physics with a model to account for the nucleon binding within the specific nucleus. The structure of the nucleon is parametrized with the three dominant form factors: two vector, $F_{1,2}\left(Q^{2}\right)$, and one axialvector, $F_{A}\left(Q^{2}\right)$. The vector form factors, including the $Q^{2}$ (squared four-momentum transfer) dependence, are welldetermined from electron scattering. The axial-vector form factor at $Q^{2}=0$ is known from neutron beta-decay. Neutrino-based CCQE measurements may then be inter- preted as a measurement of the axial-vector mass, $M_{A}$, which controls the $Q^{2}$ dependence of $F_{A}$, and ultimately, the normalization of the predicted cross section.

This simple, underlying model has led to the situation where neutrino $\mathrm{CCQE}$ measurements typically produce a measurement of $M_{A}$ independent of neutrino energy and target nucleus. The resulting world-average is $M_{A}=$ $1.03 \pm 0.02 \mathrm{GeV}[9]$ (a recent summary of the various $M_{A}$ values is provided in Ref. [10]). It should be noted that the data contributing to this world average are dominated by higher precision bubble chamber experiments using deuterium as a target. In addition, most (but not all) of the $M_{A}$ values have come from the observed distribution of CCQE events in $Q^{2}$ rather than from an overall normalization of the event yield.

Several experiments have recently reported new results on CCQE scattering from high-statistics data samples with intense, well-understood neutrino beams. The NOMAD experiment extracted a CCQE cross section and $M_{A}$ from data taken on carbon in the energy range, $3<E_{\nu}<$ $100 \mathrm{GeV}$ [10]. They observe an $M_{A}$ value and cross section consistent with the prior world-average. However, data at lower neutrino energies using carbon or oxygen as a target have indicated, through $Q^{2}$-shape fits, a somewhat larger value for $M_{A}$ (by $\approx 10 \%-30 \%$ ) [11-13]. The SciBooNE experiment has recently reported a preliminary extraction of the total CCQE cross section on carbon that is consistent with these results [14]. To add to this, the MINOS experiment has reported a preliminary result on $M_{A}$ extracted from CCQE data collected on iron in a similar energy range as NOMAD [15]. This result for $M_{A}$ is also larger than the world-average. None of these experiments has reported differential cross sections.

The CCQE cross section predictions resulting from these differing measured values for $M_{A}$ are too unreliable for use by future oscillation experiments, and the collection of existing results remains puzzling. Perhaps the currently employed model of the CCQE process is too naive and the physics of the bound nucleons can alter the extracted $M_{A}$. There is currently large theoretical interest in this area $[16,17]$ and a solution may well emerge. Regardless, if the experimental results hold up, it is clear that improved measurements will be required to sort out the situation. These measurements will need to go beyond simply extracting an $M_{A}$ value as the data are already indicating that this strategy is too simplistic. Experiments should advance to providing cross sections, differential where possible, that are correctly normalized with a predicted neutrino flux (not normalized to a different reaction channel in the same data). Experiments should also strive to reduce the model-dependence of their reported results. To this end, we present differential cross sections in muon kinematics extracted from $\nu_{\mu} \mathrm{CCQE}$ events collected in MiniBooNE.

MiniBooNE has accumulated the world's largest sample of $\nu_{\mu}$ CCQE events $(\approx 150,000)$ in the $1 \mathrm{GeV}$ region. 
Using this high-statistics and low-background event sample, we report the first measurement of an absolute $\nu_{\mu}$ CCQE double differential cross section, the main result of this work. In addition, CCQE cross sections in several other conventional forms are provided. The layout of the remainder of this paper is as follows. In Sec. II, we provide a summary of the MiniBooNE experiment, including the booster neutrino beamline (BNB) and the MiniBooNE detector. We detail the neutrino interaction model used to describe the signal and background in Sec. III. The CCQE selection and analysis strategy is outlined in Sec. IV. Finally, in Sec. V, we report the MiniBooNE fluxintegrated CCQE double differential cross section $\left(\frac{d^{2} \sigma}{d T_{\mu} d \cos \theta_{\mu}}\right)$, the flux-integrated CCQE single differential cross section $\left(\frac{d \sigma}{d Q_{\mathrm{QE}}^{2}}\right)$, and the flux-unfolded CCQE cross section as a function of energy $\left(\sigma\left[E_{\nu}^{\mathrm{QE}, \mathrm{RFG}}\right]\right)$. To facilitate comparison with updated model predictions [16,17], we provide the predicted MiniBooNE neutrino fluxes and measured cross section values in tabular form in the appendix.

\section{MINIBOONE EXPERIMENT}

\section{A. Neutrino beamline and flux}

The Booster Neutrino Beamline (BNB) consists of three major components as shown in Fig. 1: a primary proton beam, a secondary meson beam, and a tertiary neutrino beam. Protons are accelerated to $8 \mathrm{GeV}$ kinetic energy in the Fermilab Booster synchrotron and then fast-extracted in $1.6 \mu \mathrm{s}$ "spills" to the BNB. These primary protons impinge on a 1.75 interaction-length beryllium target centered in a magnetic focusing horn. The secondary mesons that are produced are then focused by a toroidal magnetic field which serves to direct the resulting beam of tertiary neutrinos towards the downstream detector. The neutrino flux is calculated at the detector with a GEANT4-based [18] simulation which takes into account proton transport to the target, interactions of protons in the target, production of mesons in the $p$-Be process, and transport of resulting particles through the horn and decay volume. A full description of the calculation with associated uncertainties is provided in Ref. [19]. MiniBooNE neutrino data
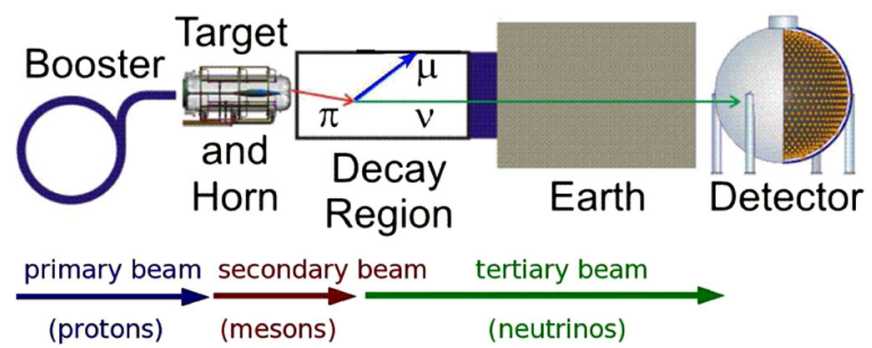

FIG. 1 (color online). Schematic overview of the MiniBooNE experiment including the booster neutrino beamline and MiniBooNE detector.
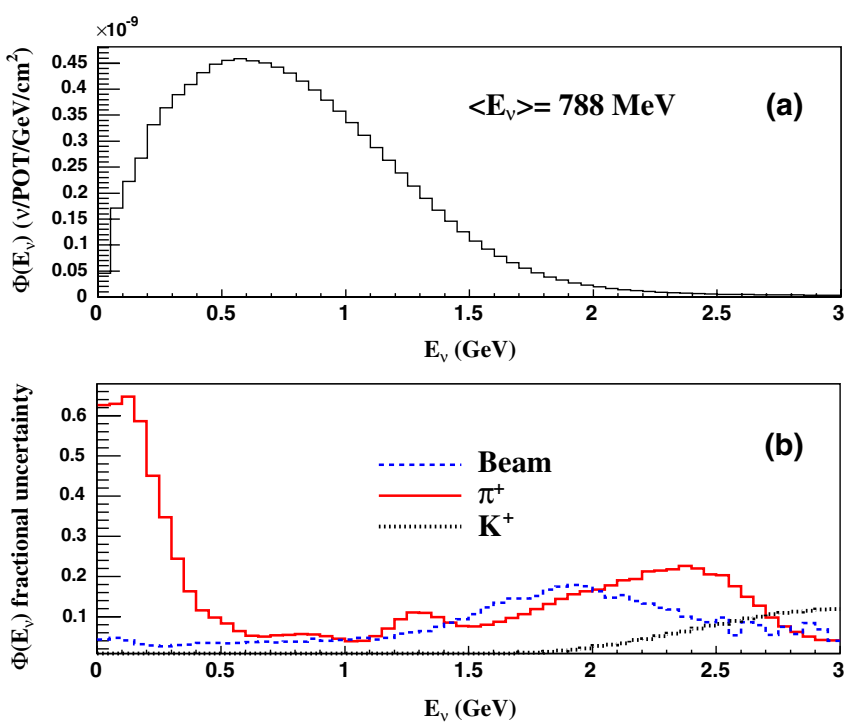

FIG. 2 (color online). Predicted $\nu_{\mu}$ flux at the MiniBooNE detector (a) along with the fractional uncertainties grouped into various contributions (b). The integrated flux is $5.16 \times$ $10^{-10} \nu_{\mu} / \mathrm{POT} / \mathrm{cm}^{2}\left(0<E_{\nu}<3 \mathrm{GeV}\right)$ with a mean energy of $788 \mathrm{MeV}$. Numerical values corresponding to the top plot are provided in Table $\mathrm{V}$ in the appendix.

is not used in any way to obtain the flux prediction. The resulting $\nu_{\mu}$ flux is shown as a function of neutrino energy in Fig. 2 along with its predicted uncertainty. These values are tabulated in Table $\mathrm{V}$ in the appendix. The $\nu_{\mu}$ flux has an average energy (over $0<E_{\nu}<3 \mathrm{GeV}$ ) of $788 \mathrm{MeV}$ and comprises $93.6 \%$ of the total flux of neutrinos at the MiniBooNE detector. There is a 5.9\% (0.5\%) contamination of $\bar{\nu}_{\mu}\left(\nu_{e}, \bar{\nu}_{e}\right)$; all events from these (non- $\left.\nu_{\mu}\right)$ neutrino types are treated as background in this measurement (Sec. IV D).

The largest error on the predicted neutrino flux results from the uncertainty of pion production in the initial $p$-Be process in the target as the simulation predicts that $96.7 \%$ of muon neutrinos in the BNB are produced via $\pi^{+}$decay. The meson production model in the neutrino beam simulation [19] relies on external hadron production measurements. Those of the HARP experiment [20] are the most relevant as they measure the $\pi^{ \pm}$differential cross section in $p$-Be interactions at the same proton energy and on the same target material as MiniBooNE. The uncertainty in $\pi^{+}$production is determined from spline fits to the HARP $\pi^{+}$double differential cross section data [19]. The splinefit procedure more accurately quantifies the uncertainty in the underlying data, removing unnecessary sources of error resulting from an inadequate parameterization [21] of the HARP data. The HARP data used was that from a thin $(5 \%$ interaction length) beryllium target run [20]. While that data provides a valuable constraint on the BNB flux prediction, additional uncertainties resulting from thick target effects (secondary rescattering of protons and pions) are included through the BNB flux simulation. 
The resulting $\pi^{+}$production uncertainty is $\approx 5 \%$ at the peak of the flux distribution increasing significantly at high and low neutrino energies. There is a small contribution to the $\nu_{\mu}$ flux error from the uncertainty in kaon production which is significant only for $E_{\nu}>2.0 \mathrm{GeV}$. Other major contributions to the flux error include uncertainties on the number of protons on target (POT), hadron interactions in the target, and the horn magnetic field. These are grouped as the "beam" component shown together with the aforementioned pion and kaon production uncertainties in Fig. 2(b). All flux errors are modeled through variations in the simulation and result in a total error of $\approx 9 \%$ at the peak of the flux. In practice, a complete error matrix is calculated in bins of neutrino energy that includes correlations between bins. This matrix is used to propagate the flux uncertainties to the final quantities used to extract the cross section results reported here.

\section{B. MiniBooNE detector}

The MiniBooNE detector (shown schematically in Fig. 1) is located $541 \mathrm{~m}$ downstream of the neutrino production target and consists of a spherical steel tank of $610 \mathrm{~cm}$ inner radius filled with 818 tons of Marcol7 light mineral oil $\left(\mathrm{CH}_{2}\right)$ with a density of $0.845 \mathrm{~g} / \mathrm{cm}^{3}$. The volume of the tank is separated into an inner and an outer region via an optical barrier located at a radius of $574.6 \mathrm{~cm}$. The inner and outer regions are only separated optically, the oil is the same in each. The index of refraction of the oil is 1.47 , yielding a Cherenkov threshold for particles with $\beta>0.68$. The mineral oil is undoped, that is, no additional scintillation solutes were added. However, because of intrinsic impurities in the oil, it produces a small amount of scintillation light in addition to Cherenkov light in response to energy loss by charged particles.

The inner (signal) region is viewed by an array of 1280 inward-facing 8-inch photo multiplier tubes (PMTs) mounted on the inside of the optical barrier and providing $11.3 \%$ photocathode coverage of the surface of the inner tank region. The outer (veto) region is monitored by 240 pair-mounted PMTs which record the light produced by charged particles entering or exiting the detector volume.

The PMT signals, in response to the light produced from charged particles, are routed to custom-built electronics modules where they are amplified, discriminated, and digitized. These ("QT") modules extract the start time and integrated charge from each PMT pulse that is above a discriminator threshold of $\approx 0.1$ photoelectron. This unit of data is called a PMT "hit." The data is stored in a temporary buffer until a trigger decision is made. The trigger system uses information from the Fermilab accelerator clock signals and PMT multiplicities to form physics and calibration trigger signals. The physics trigger for this analysis requires only that beam be sent to the BNB neutrino production target. When this condition is satisfied, all PMT-hit data in a $19.2 \mu$ s window starting $5 \mu$ s before the $1.6 \mu \mathrm{s}$ beam spill is extracted from the QT modules and added to the data stream. This readout strategy collects all PMT data (with no multiplicity threshold) from beaminduced neutrino events as well as any muon decays that occur with a characteristic time of $2 \mu$ s after the neutrino interaction.

The data within the $19.2 \mu$ s readout window is examined at the analysis stage to organize the hits into temporal clusters or "subevents." A subevent is any group of at least 10 hits (from inner or outer PMTs) where no two consecutive hits are separated in time by more than $10 \mathrm{~ns}$. These subevents may then be analyzed separately to extract further information such as energy and position. With this scheme, muon-decay electrons or positrons may be identified and separated from the primary neutrino event.

The MiniBooNE detector is calibrated via the light from a pulsed laser source, cosmic muons, and muon-decay electrons. The laser calibration system consists of a pulsed diode laser injecting light via optical fibers into four $10 \mathrm{~cm}$ dispersion flasks located at various depths in the main detector volume. In addition, one bare fiber is installed at the top of the tank. This system is used to quantify the charge and time response of the PMTs and allows for an in situ measurement of the oil attenuation length and light scattering properties.

The muon calibration system consists of a two-layer scintillation hodoscope located above the detector tank combined with seven 5-cm cubic scintillators deployed at various locations within the tank (near the vertical axis) and used to tag stopped muons. With this system, stopping cosmic ray muons of energies ranging from 20 to $800 \mathrm{MeV}$ are tracked through the detector enabling an energy calibration via the known range of the stopping muons. Using this data, the energy (angular) resolution for reconstructed muons in MiniBooNE is measured to be $12 \%\left(5.4^{\circ}\right)$ at $100 \mathrm{MeV}$, improving to $3.4 \%\left(1.0^{\circ}\right)$ at $800 \mathrm{MeV}$.

A separate large sample of stopping cosmic muons is obtained via a dedicated calibration trigger that requires the signature of an incoming muon and its decay electron. This sample allows for the calibration and measurement of the detector response to muon-decay electrons.

More details about the oil medium, detector structure, PMTs, electronics, and calibration are available in Ref. [22].

\section{Detector simulation}

A GEANT3-based [23] program is used to simulate the response of the detector to neutrino interactions. This simulation is used to determine the detection efficiency for CCQE events, the probability for accepting background events, and the error on relevant observables due to uncertainties in the detector response.

The entire geometry of the MiniBooNE detector is modeled including the detector tank and all inner compo- 
nents. The major components of the detector housing structure are modeled as is the surrounding environment. Standard GEANT3 particle propagation and decay routines are utilized with some changes made to better simulate $\pi^{0}$ decay, $\mu$ decay, and $\mu^{-}$capture on carbon. The latter process is especially important for the background estimation in the analysis reported here. The GCALOR [24] hadronic interaction package is used in place of the default GFLUKA package in GEANT3 because it better reproduces known data on $\pi^{+}$absorption and charge exchange in the relevant $\pi^{+}$energy range (100-500 MeV).

Some modifications were made to the standard GCALOR code to better simulate $\pi^{ \pm}$processes. Pion radiative capture/decay and photonuclear processes are of concern for the neutrino oscillation search [2] but have negligible effect for this analysis. The elastic scattering of $\pi^{ \pm}$on carbon is important and was not simulated in the standard GCALOR code. A model, guided by the available data $[25,26]$, was added to the simulation and yields only a small change to the calculated background from pion production processes.

Uncertainties of $35 \%$ on $\pi^{+}$absorption and $50 \%$ on $\pi^{+}$ charge exchange are assigned based on the difference between the external data [25] and the GCALOR prediction. Note that these errors are relevant for $\pi^{+}$propagation in the detector medium not intranuclear processes which are assigned separate uncertainties (Sec. III D).

Charged particles propagating through the detector oil produce optical photons via Cherenkov radiation and scintillation. Optical wavelengths of $250-650 \mathrm{~nm}$ are treated. The Cherenkov process is modeled with standard GEANT3. The scintillation process is modeled with a MiniBooNE-specific simulation that creates optical photons at a rate proportional to Birk's law-corrected energy loss with an emission spectrum determined from dedicated florescence measurements. Optical photons resulting from these production processes are tracked through the detector oil with consideration of scattering, fluorescence, absorption, and reflection from detector surfaces (including PMTs). Photons that intersect the PMT surface (and do not reflect) are modeled with a wavelength and incident angle-dependent efficiency. The photon signal in each PMT is used together with the known response of the PMT and readout electronics to generate simulated data that is then input to the data analysis programs.

The models, associated parameters, and errors implemented in the detector simulation are determined through external measurements of the properties of materials as well as internal measurements using data collected in the MiniBooNE detector. It is particularly important to correctly model the optical photon transport since a typical optical photon travels several meters before detection. This "optical model" is tuned starting from various external (to the detector) measurements of MiniBooNE mineral oil optical properties, such as the refractive index, attenuation length, and fluorescence/scintillation strength. These quantities allow for the implementation of various models to describe the optical photon propagation. The details of the models are then further adjusted based on MiniBooNE internal data sets such as muon-decay electrons, cosmic muons, and laser pulses. In total, 35 optical model parameters are adjusted to obtain a good description of the various data sets. Values for the uncertainties of these parameters, including correlations among them, are also extracted from the data and the effect on the reported observables determined by running the simulation with adjusted values (Sec. IV E).

Additional details about the MiniBooNE detector simulation and supporting measurements of oil properties are available in Refs. [22,27].

\section{NEUTRINO INTERACTION MODEL}

The MiniBooNE experiment employs the NUANCE v3 event generator [28] to estimate neutrino interaction rates in the $\mathrm{CH}_{2}$ target medium. The NUANCE generator considers all interaction processes possible in the neutrino energy region relevant for MiniBooNE. It also enables the various processes to be tuned to match the data via input parameters or source code changes where necessary. The NUANCE generator includes the following components: (1) a relativistic Fermi gas (RFG) model for CCQE (and NC elastic) scattering on carbon [8]; (2) a baryonic resonance model for $\mathrm{CC} / \mathrm{NC}$ single and multipion production [29]; (3) a coherent CC/NC single pion production model [30]; (4) a deep inelastic scattering (DIS) model [31,32]; and (5) a final-state interaction model to simulate reinteraction of final state hadrons in the nuclear medium [28]. Neutrino interactions on both free (protons) and bound nucleons (in carbon) are considered to model the $\mathrm{CH}_{2}$ detector medium. Further details on these models, parameters, and uncertainties are provided in the following subsections.

This event generator is used, after the adjustment of parameters to adequately describe the MiniBooNE data, to calculate the background contribution to the CCQE signal. The CCQE model is used only in the extraction of the model parameters in a shape-fit to the $Q_{\mathrm{QE}}^{2}$ distribution (Sec. IV C). The CCQE differential cross section measurements do not depend on this model (excepting some small dependence due to detector resolution corrections and $\bar{\nu}_{\mu}$ CCQE backgrounds). In addition, since it is such a large background to $\mathrm{CCQE}$, the $\mathrm{CC} 1 \pi^{+}$background is constrained (outside of the NUANCE model) to reproduce MiniBooNE data (Sec. IV C). A summary of interaction channels considered and the NUANCE predictions for the neutrino interaction fractions in the MiniBooNE detector are provided in Table I. The final values for the predicted backgrounds (after event selection cuts) are provided in Table III. 
TABLE I. Event type nomenclature and NUANCE-predicted $\nu_{\mu}$ event fractions for MiniBooNE integrated over the predicted flux in neutrino mode before selection cuts. For the pion production channels, indirect production (through resonance states) and direct production (through coherent processes) are included. $(\mathrm{CC}=$ charged-current, $\mathrm{NC}=$ neutral-current $)$.

\begin{tabular}{lccc}
\hline \hline neutrino process & abbreviation & reaction & fraction $(\%)$ \\
\hline CC quasielastic & $\mathrm{CCQE}$ & $\nu_{\mu}+n \rightarrow \mu^{-}+p$ & 39 \\
$\mathrm{NC}$ elastic & $\mathrm{NCE}$ & $\nu_{\mu}+p(n) \rightarrow \nu_{\mu}+p(n)$ & 16 \\
$\mathrm{CC} 1 \pi^{+}$production & $\mathrm{CC} 1 \pi^{+}$ & $\nu_{\mu}+p(n) \rightarrow \mu^{-}+\pi^{+}+p(n)$ & 25 \\
$\mathrm{CC} 1 \pi^{0}$ production & $\mathrm{CC} 1 \pi^{0}$ & $\nu_{\mu}+n \rightarrow \mu^{-}+\pi^{0}+p$ & 4 \\
$\mathrm{NC} 1 \pi^{ \pm}$production & $\mathrm{NC} 1 \pi^{ \pm}$ & $\nu_{\mu}+p(n) \rightarrow \nu_{\mu}+\pi^{+}\left(\pi^{-}\right)+n(p)$ & 4 \\
$\mathrm{NC} 1 \pi^{0}$ production & $\mathrm{NC} 1 \pi^{0}$ & $\nu_{\mu}+p(n) \rightarrow \nu_{\mu}+\pi^{0}+p(n)$ & 8 \\
multipion production, DIS, etc. & other & $\nu_{\mu}+p(n) \rightarrow \mu^{-}+N \pi^{ \pm}+X$, etc. & 4 \\
\hline \hline
\end{tabular}

\section{A. Charged current quasielastic scattering}

CCQE scattering is the dominant neutrino interaction process in MiniBooNE and the subject of this analysis. This process is defined as the charge-changing scattering of a neutrino from a single nucleon with no other particles produced and it is simulated with the RFG model [8] with several modifications. A dipole form is used for the axial form factor with an adjustable axial mass, $M_{A}$. An empirical Pauli-blocking parameter, $\kappa$, is introduced [11] to allow for an extra degree of freedom that is important to describe the MiniBooNE data at low momentum transfer. This parameter is a simple scaling of the lower bound, $E_{\mathrm{lo}}$, of the nucleon energy integral of Ref. [8] via $E_{\mathrm{lo}}=$ $\kappa\left(\sqrt{p_{F}^{2}+M_{p}^{2}}-\omega+E_{B}\right)$, where $p_{F}, M_{p}, \omega$, and $E_{B}$ are the Fermi momentum, nucleon mass, energy transfer, and binding energy, respectively. When $\kappa>1$, the Pauliblocking of final-state nucleons is increased which reduces the cross section at low momentum transfer.

A parametrization [33] is used to describe the nondipole behavior of the Dirac and Pauli form factors. Although the contribution is small, the pseudoscalar form factor, derived from partial conservation of the axial-vector current (PCAC) is also included [34]. The scalar and axial tensor form factors are set to zero as implied from G-parity conservation. The Fermi momentum and binding energy for carbon are set to $220 \pm 30 \mathrm{MeV} / \mathrm{c}$ and $34 \pm 9 \mathrm{MeV}$, respectively, as extracted from electron scattering data [35] taking account of the purely isovector character of CCQE.

The parameters $M_{A}$ and $\kappa$ were initially extracted from MiniBooNE CCQE data in a prior analysis [11], and were determined to be $M_{A}^{\text {eff }}=1.23 \pm 0.20 \mathrm{GeV}$ and $\kappa=$ $1.019 \pm 0.011$. While not the main result of this paper, this exercise is repeated after explicitly measuring the $\mathrm{CC} 1 \pi^{+}$background from MiniBooNE data and is described in Sect. IVC. The superscript "eff" on $M_{A}$ was introduced to allow for the possibility that the axial mass measured from scattering on nucleons bound in carbon may be different from the "bare-nucleon" axial mass that appears within the neutrino model. The use of this notation is continued in this work. The uncertainties in these CCQE model parameters do not propagate to the errors on the measured cross sections for this channel. Neutral current elastic (NCE) scattering is described with the same model as that for the CCQE interaction with the replacement of appropriate form factors [36] to describe the NC coupling to the nucleon. The uncertainty from the NCE model parameters on the CCQE results is negligible due to the small background contribution from this channel (Table III).

\section{B. Resonance interactions}

The primary source of single pion production for MiniBooNE is predicted to be baryonic resonance production and decay, such as,

$$
\begin{aligned}
\nu_{\mu}+p \rightarrow \mu^{-}+ & \Delta^{++} \\
& \hookrightarrow \pi^{+}+p, \\
\nu_{\mu}+n \rightarrow \mu^{-}+ & \Delta^{+} \\
& \hookrightarrow \pi^{+}+n, \quad \pi^{0}+p .
\end{aligned}
$$

The NUANCE model employs the relativistic harmonic oscillator quark model of Refs. [29,37]. The pion angular distribution due to the spin structure of the resonance states is additionally taken into account. In total, 18 resonances and their interferences are simulated in reactions with invariant mass $W<2 \mathrm{GeV}$, however, the $\Delta(1232)$ resonance dominates at this energy scale. For reactions on bound nucleons, an RFG model is employed with a uniform Fermi momentum and constant binding energy. Inmedium effects on the width of resonances are not considered explicitly, however, final-state interactions can produce an effective change in these widths. An axial mass of $M_{A}^{1 \pi}=1.10 \pm 0.27 \mathrm{GeV}$, set by tuning to available data, is used for this channel.

Multipion processes are considered in the NUANCE simulation with $M_{A}^{N \pi}=1.30 \pm 0.52 \mathrm{GeV}$. This parameter was set (together with $M_{A}^{1 \pi}$ ) so that the simulation reproduces inclusive CC data. The contribution of this channel to the CCQE background is small and the uncertainty is negligible in the final errors. 
The $\mathrm{CC} 1 \pi^{+}$channel is the largest background contribution to the CCQE signal and the uncertainty from the model prediction alone is substantial [38]. However, the experimental signature of the $\mathrm{CC} 1 \pi^{+}$reaction in MiniBooNE is distinct and the efficiency is large. So, in order to reduce uncertainty stemming from the $\mathrm{CC} 1 \pi^{+}$ model prediction, a measurement of the $\mathrm{CC} 1 \pi^{+}$background is performed as part of the CCQE analysis (Sec. IV C).

\section{Coherent pion production}

Pions are also produced in the $\mathrm{CC}$ and $\mathrm{NC}$ coherent interaction of neutrinos with carbon nuclei,

$\nu_{\mu}+A \rightarrow \mu^{-}+\pi^{+}+A, \quad \nu_{\mu}+A \rightarrow \nu_{\mu}+\pi^{0}+A$.

In NUANCE, this coherent pion production process is described using the model of Ref. [30] assuming $M_{A}^{\text {coh }}=$ $1.03 \pm 0.28 \mathrm{GeV}[28]$.

Coherent scattering is predicted to have distinct features in the angular distributions of both the final-state muons and pions. Both the K2K [39] and MiniBooNE [40] experiments have measured the fraction of pions produced coherently in $\approx 1 \mathrm{GeV}$ neutrino interactions. $\mathrm{K} 2 \mathrm{~K}$ measured a rate for coherent $\mathrm{CC} 1 \pi^{+}$production consistent with zero and set an upper limit. MiniBooNE measured a nonzero value for coherent $\mathrm{NC} 1 \pi^{0}$ production albeit $\approx 35 \%$ smaller than the model prediction $[28,30]$. The latest result from the SciBooNE experiment is consistent with the $\mathrm{K} 2 \mathrm{~K}$ measurement for $\mathrm{CC} 1 \pi^{+}$coherent production [41].

Because of the current discrepancy between CC and NC coherent pion measurements and the variation in model predictions at low energy, the prediction is reduced to $50 \%$ of the default value in NUANCE $[28,30]$ and assigned a $100 \%$ uncertainty. This choice spans the current results from relevant experiments and existing theoretical predictions.

\section{Final state interactions}

In the NUANCE simulation, neutrino interactions on nucleons are modeled using the impulse approximation which assumes the interaction occurs instantaneously on independent nucleons. The binding of nucleons within carbon is treated within the RFG model, however, any nucleon-nucleon correlation effects are not. The final-state hadrons may interact within the nucleus as they exit. They are propagated through the ${ }^{12} \mathrm{C}$ nucleus with a known, radially-dependent nucleon density distribution [42] and may undergo final-state interactions (FSI). These are simulated by calculating interaction probabilities for the possible processes in $0.3 \mathrm{fm}$ steps until the particles leave the $\approx 2.5 \mathrm{fm}$-radius spherical carbon atom. The interaction probabilities are derived from external $\pi-N, N-N$ cross section and angular distribution data [43], as well as the nuclear density of carbon.
To model $\Delta$ absorption in the nucleus, $(\Delta+N \rightarrow N+$ $N$ ), a constant, energy-independent probability for an intranuclear interaction of 20\% (10\%) is assumed for $\Delta^{+}+N$, $\Delta^{0}+N\left(\Delta^{++}+N, \Delta^{-}+N\right)$ processes. These values were chosen based on comparisons to K2K data [44] and are assigned a $100 \%$ uncertainty. After an interaction, the density distribution and step size are modified to prevent an overestimate of these FSI effects $[45,46]$.

Of these hadron FSI processes, intranuclear pion absorption and pion charge exchange, $\left(\pi^{+}+X \rightarrow X^{\prime}, \pi^{+}+\right.$ $X \rightarrow \pi^{0}+X^{\prime}$ ) are the most important contributions to the uncertainty in the CCQE analysis. Pion absorption and charge exchange in the detector medium are addressed separately in the detector simulation (Sec. II C).

A $\mathrm{CC} 1 \pi^{+}$interaction followed by intranuclear pion absorption is effectively indistinguishable from the CCQE process in MiniBooNE (they are "CCQE-like," Sec. IV D). An event with intranuclear pion charge exchange is distinguishable in the detector, albeit not with $100 \%$ efficiency. These effects, combined with the high rate of $\mathrm{CC} 1 \pi^{+}$events, results in a significant background to the CCQE measurement that must be treated carefully. The model for these intranuclear pion processes has been tuned to match the available data [25] in the relevant pion energy range. A comparison of the adjusted NUANCE model and relevant data is shown in Fig. 3. A 25\% (30\%) systematic error in the overall interaction cross section is used for the pion absorption (charge-exchange) process.
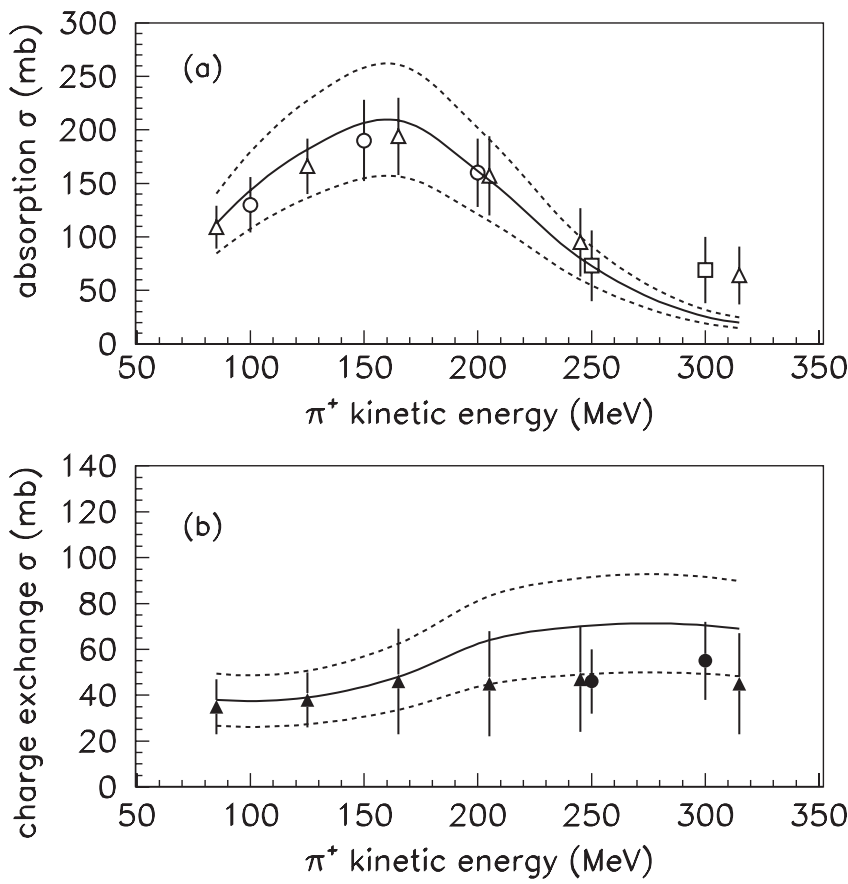

FIG. 3. A comparison of relevant data [25] with the NUANCE model (solid lines) for intranuclear pion (a) absorption and (b) charge exchange as a function of pion kinetic energy. The dotted lines show the $25 \%$ (30\%) systematic error bands assumed for the pion absorption (charge-exchange) cross sections. 


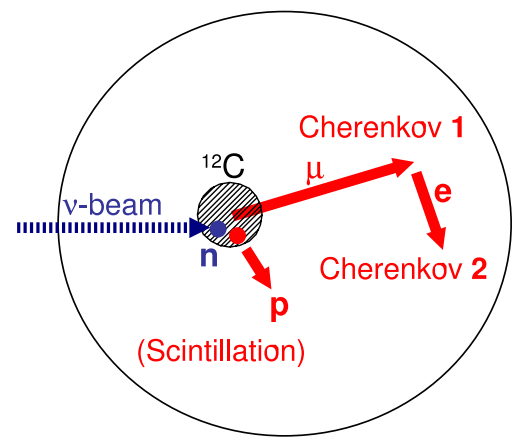

FIG. 4 (color online). Schematic illustration of a CCQE interaction in the MiniBooNE detector. The primary Cherenkov light from the muon (Cherenkov 1, first subevent) and subsequent Cherenkov light from the decay electron (Cherenkov 2, second subevent) are used to tag the CCQE event. No requirements are made on the outgoing proton.

\section{CCQE MEASUREMENT}

The goal of this measurement is to determine the double differential cross section for the CCQE process on carbon, $\nu_{\mu}+n \rightarrow \mu^{-}+p$, where the target neutron is bound in ${ }^{12} \mathrm{C}$.

The identification of CCQE interactions in the MiniBooNE detector relies solely on the detection of the Cherenkov light from the primary (prompt) muon and the associated decay-electron. An illustration of this process is shown in Fig. 4. Scintillation light is produced by the charged lepton and the recoil proton (or nuclear fragments). However, with the reconstruction employed here, this light is not separable from the dominant Cherenkov light. In addition, the proton is typically below Cherenkov threshold. These conditions are such that the proton is not separable from the charged lepton and so no requirement is placed on the recoil proton in this analysis. This is to be contrasted with some measurements of CCQE interactions that do require the observation of a recoil proton for some part of the event sample [10,12-14]. An advantage of this insensitivity to the proton recoil is that the extracted cross sections are less dependent on proton final-state model uncertainties. However, the disadvantage in not detecting the recoil nucleon is that contributions to scattering from other nuclear configurations (such as two-nucleon correlations) are inseparable. These contributions are, in the strictest sense, not CCQE, but counted as such in our experimental definition.

A requirement of low veto activity for the CCQE sample ensures that all particles produced in the event stop in the main region of the detector. This allows muons to be tagged with high efficiency via their characteristic electron-decay with $\tau \approx 2 \mu \mathrm{s}$.

The CCQE interaction, including the muon decay, proceeds as

$$
\begin{array}{ll}
\text { 1: } \nu_{\mu}+n \rightarrow & \mu^{-}+p \\
2: & \hookrightarrow e^{-}+\bar{\nu}_{e}+\nu_{\mu} .
\end{array}
$$

where each line in this equation identifies a subevent (Sec. IIB). The primary muon is identified with the first subevent and the subsequent decay-electron with the second subevent. At BNB neutrino energies, neutrino interaction events that contain a primary muon predominantly result from CCQE scattering as can be seen in Table I.

The largest background is from $\mathrm{CC}$ single-pion production $\left(\mathrm{CC} 1 \pi^{+}\right)$. A CC $1 \pi^{+}$interaction in the detector consists of (with subevents labeled),

$$
\begin{array}{ccc}
\text { 1: } \nu_{\mu}+p(n) \rightarrow & \mu^{-}+p(n)+\pi^{+} & \\
& \hookrightarrow \mu^{+}+\nu_{\mu} \\
\text { 2: } & \hookrightarrow e^{-}+\bar{\nu}_{e}+\nu_{\mu} & \hookrightarrow e^{+}+\nu_{e}+\bar{\nu}_{\mu} .
\end{array}
$$

Note that this interaction results in three subevents: the primary interaction and two muon decays (the muon decays can occur in any order). The $\pi^{+}$decays immediately and light from the prompt decay products contribute to the total light in the primary event. These events may be removed from the CCQE sample by requiring exactly two subevents. This requirement also reduces the background from $\mathrm{NC}$ processes to an almost negligible level because they do not contain muons and thus have only one subevent. This simple strategy results in a fairly pure sample of CCQE events. However, a significant number of $\mathrm{CC} 1 \pi^{+}$events have only two subevents because one of the decay electrons escapes detection: the $\mu^{-}$is captured on ${ }^{12} \mathrm{C}$ in the mineral oil (with $8 \%$ probability [47]) or the $\pi^{+}$is absorbed. Additionally, the study of $\mathrm{CC} 1 \pi^{+}$events for this analysis has indicated that the prediction for the $\mathrm{CC} 1 \pi^{+}$channel from the NUANCE event generator is not sufficiently accurate for this measurement [38]. For these reasons, the $\mathrm{CC} 1 \pi^{+}$rate is measured using a dedicated event sample. This differs from our previous strategy [11] where the default NUANCE-predicted $\mathrm{CC} 1 \pi^{+}$fraction (with no adjustments) was used, with generous errors, in fits to the CCQE sample.

The resulting procedure for selecting the CCQE sample and measuring the $\mathrm{CC} 1 \pi^{+}$background involves the following steps:

(1) selection of a "supersample" of events with a clean muon signature to isolate $\mathrm{CC}$ events (predominantly CCQE and $\mathrm{CC} 1 \pi^{+}$) via analysis cuts;

(2) application of a subevent cut to separate the supersample into CCQE (2-subevents) and $\mathrm{CC} 1 \pi^{+}$(3subevents) samples;

(3) measurement of the $\mathrm{CC} 1 \pi^{+}$rate from the $\mathrm{CC} 1 \pi^{+}$ sample;

(4) adjustment of the $\mathrm{CC} 1 \pi^{+}$model in the event simulation to reproduce the measured rate; and 
(5) subtraction of this adjusted $\mathrm{CC} 1 \pi^{+}$background (along with other predicted backgrounds) from the CCQE signal to produce a a measurement of the CCQE interaction cross section.

The details of this procedure are provided in the following subsections.

In this analysis, the reconstruction of the $\mathrm{CC} 1 \pi^{+}$sample is for the sole purpose of background estimation. Dedicated measurements of the $\mathrm{CC} 1 \pi^{+}$and $\mathrm{CC} 1 \pi^{0}$ channels in MiniBooNE have been reported elsewhere [48-50] including detailed reconstruction of the $\pi^{+}$and $\pi^{0}$ kinematics.

\section{A. Event reconstruction}

For this analysis, it is crucial to identify and measure the muon in the CC interaction. This is accomplished with an "extended-track" reconstruction algorithm [51] which uses the charge and time information from all PMT hits in the first subevent to form a likelihood that is maximized to determine the best single track hypothesis quantified by the track starting point, starting time, direction, and kinetic energy. This is performed with both a muon and electron particle hypothesis from which a $(\log )$ likelihood ratio is formed to enable particle identification.

The muon kinetic energy $T_{\mu}$ and muon scattering angle $\theta_{\mu}$ are extracted from the track reconstruction assuming a muon hypothesis. These are used to form the fundamental observable reported here, the double differential cross section. For additional reported observables, the reconstructed neutrino energy $E_{\nu}^{\mathrm{QE}}$ and reconstructed fourmomentum transfer $Q_{\mathrm{QE}}^{2}$ are obtained via,

$$
\begin{gathered}
E_{\nu}^{\mathrm{QE}}=\frac{2\left(M_{n}^{\prime}\right) E_{\mu}-\left(\left(M_{n}^{\prime}\right)^{2}+m_{\mu}^{2}-M_{p}^{2}\right)}{2 \cdot\left[\left(M_{n}^{\prime}\right)-E_{\mu}+\sqrt{E_{\mu}^{2}-m_{\mu}^{2}} \cos \theta_{\mu}\right]}, \\
Q_{\mathrm{QE}}^{2}=-m_{\mu}^{2}+2 E_{\nu}^{\mathrm{QE}}\left(E_{\mu}-\sqrt{E_{\mu}^{2}-m_{\mu}^{2}} \cos \theta_{\mu}\right),
\end{gathered}
$$

where $E_{\mu}=T_{\mu}+m_{\mu}$ is the total muon energy and $M_{n}$, $M_{p}, m_{\mu}$ are the neutron, proton, and muon masses. The adjusted neutron mass, $M_{n}^{\prime}=M_{n}-E_{B}$, depends on the binding energy (or more carefully stated, the separation energy) in carbon, $E_{B}$, which for this analysis is set to $34 \pm$ $9 \mathrm{MeV}$.

The subscript, "QE," on these reconstructed quantities is to call attention to these specific definitions and to distinguish them from quantities obtained in other ways such as fits to the underlying true kinematic quantities. These are kinematic definitions that assume the initial nucleon (neutron) is at rest and the interaction is CCQE ("QE assumption"). While these quantities certainly differ from the underlying true quantities, they are well-defined, unambiguous, and easily reproduced by others.

\section{B. CCQE and $\mathrm{CC} 1 \pi^{+}$event selection}

The CCQE and $\mathrm{CC} 1 \pi^{+}$candidate events are selected for this analysis and separated with a sequence of cuts summarized in Table II.

The first five cuts are designed to efficiently select a high-purity sample of CCQE and $\mathrm{CC} 1 \pi^{+}$events. Cut 1 rejects events with incoming particles such as cosmic rays or neutrino-induced events produced in the surrounding material. It also eliminates events where any of the neutrino interaction products escape the main detector volume. This is important for an accurate muon energy measurement and to avoid missing muon decays which leads to higher backgrounds. Cut 1 does reduce the efficiency substantially (Table II), however, it is necessary to reduce background (together with the subsequent cuts). Cut 2 requires that the primary (muon) is in-time with the BNB spill window. Cut 3 ensures that the reconstructed primary muon vertex is located within a fiducial region in the main detector volume sufficiently far from the PMTs for accurate reconstruction. Cut 4 provides a minimum muon kinetic energy for reliable reconstruction and reduces backgrounds from beam-unrelated muon-decay electrons.

Cut 5 requires that the candidate primary muon is better fit as a muon than as an electron. Misreconstructed and multiparticle events tend to prefer the electron hypothesis so this cut reduces such contamination. This also substantially reduces the efficiency for selecting $\mathrm{CC} 1 \pi^{+}$events as

TABLE II. List of cuts for the CCQE and CC1 $\pi^{+}$event selections. The predicted efficiency and purity values are for the CCQE

\begin{tabular}{|c|c|c|c|}
\hline \multirow[b]{2}{*}{ cut \# } & \multirow[b]{2}{*}{ description } & \multicolumn{2}{|c|}{ CCQE } \\
\hline & & effic. $(\%)$ & purity $(\%)$ \\
\hline 1 & all subevents, \# of veto hits $<6$ & 54.8 & 36.8 \\
\hline 2 & 1st subevent, event time window, $4400<T$ (ns) $<6400$ & 54.3 & 36.8 \\
\hline 3 & 1 st subevent, reconstructed vertex radius $<500 \mathrm{~cm}$ & 45.0 & 37.4 \\
\hline 4 & 1st subevent, kinetic energy $>200 \mathrm{MeV}$ & 39.7 & 46.3 \\
\hline 5 & 1st subevent, $\mu / e$ log-likelihood ratio $>0.0$ & 36.0 & 62.3 \\
\hline 6 & $\#$ total subevents $=2$ for $\mathrm{CCQE}\left(=3\right.$ for $\left.\mathrm{CC} 1 \pi^{+}\right)$ & 29.1 & 71.0 \\
\hline \multirow[t]{2}{*}{7} & (CCQE-only) 1 st subevent, $\mu-e$ vertex distance $>100 \mathrm{~cm}$ and & & \\
\hline & $\mu-e$ vertex distance $>\left(500 \times T_{\mu}(\mathrm{GeV})-100\right) \mathrm{cm}$ & 26.6 & 77.0 \\
\hline
\end{tabular}
signal normalized to all CCQE events with a reconstructed vertex radius, $r<550 \mathrm{~cm}$. 

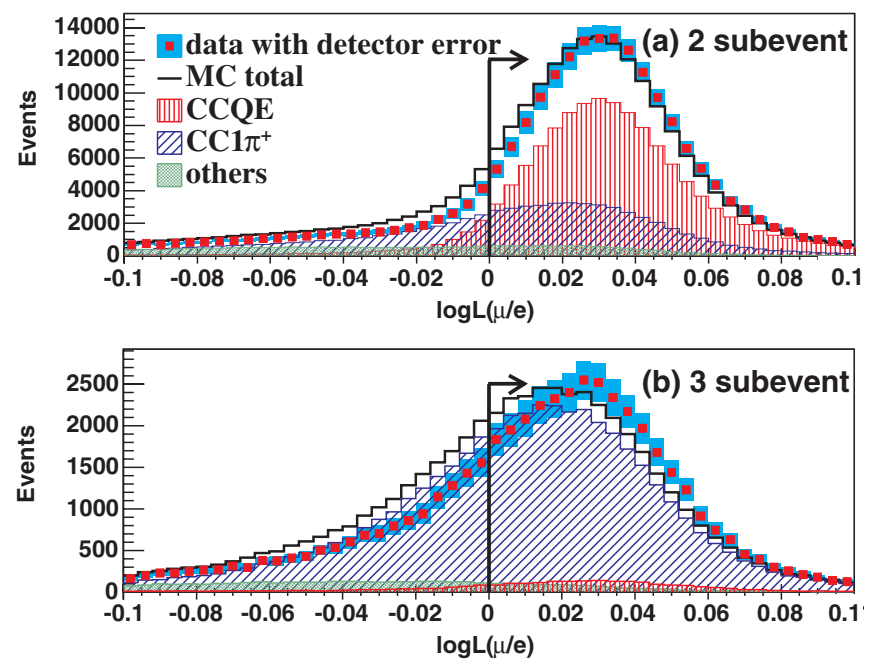

FIG. 5 (color online). Distributions of the $\mu / e$ log-likelihood ratio for the (a) 2 and (b) 3 subevent samples. Data and Monte Carlo simulation (MC) are shown along with the individual MC contributions from CCQE, $\mathrm{CC} 1 \pi^{+}$, and other channels. The lines with arrows indicate events selected by the muonelectron likelihood ratio cut.

can be seen in Fig. 5 where the $\mu / e$ log-likelihood ratio distribution is shown for each of the 2- and 3-subevent samples. This bias is intended as it selects a sample of $\mathrm{CC} 1 \pi^{+}$with muon kinematics more closely matched to those $\mathrm{CC} 1 \pi^{+}$that are background to the CCQE sample. As is shown in Fig. 5, data and Monte Carlo simulation (MC) agree fairly well to within the detector errors. The loglikelihood ratio distribution is quite sensitive to details of an event such as scintillation from hadron recoil via the PMT charge and time information [51]. The data-MC difference in the number of events passing Cut 5 in both the 2- and 3-subevent samples is covered by the full systematic errors considered in this analysis.

Cut 6 separates the samples into CCQE (2 subevents) and $\mathrm{CC} 1 \pi^{+}$( 3 subevents) candidates. For this analysis, the second and third subevents are required to contain at least 20 tank hits to reduce the probability of accidental coincidences with the initial neutrino interaction (first subevent). This requirement reduces the efficiency for identifying the muon-decay electron by $\approx 3 \%$.

Cut 7 utilizes the muon-electron vertex distance, the measured separation between the reconstructed muon and electron vertices. This cut requires that the decay-electron is correctly associated with the primary muon and is applied to the CCQE (2-subevent) sample only. This eliminates many $\mathrm{CC} 1 \pi^{+}$events where the second subevent is a decay-positron from the $\pi^{+}$decay chain and not the electron from the decay of the primary muon. The distributions of the muon-electron vertex distance for the major Monte Carlo channels and for data are shown in Fig. 6, after the application of cuts 1-6 for events with 2 subevents.

As shown in Table II, the efficiency for finding CCQE events with a reconstructed vertex radius, $r<550 \mathrm{~cm}$, is $26.6 \%$. An $r<550 \mathrm{~cm}$ volume is used for normalization in
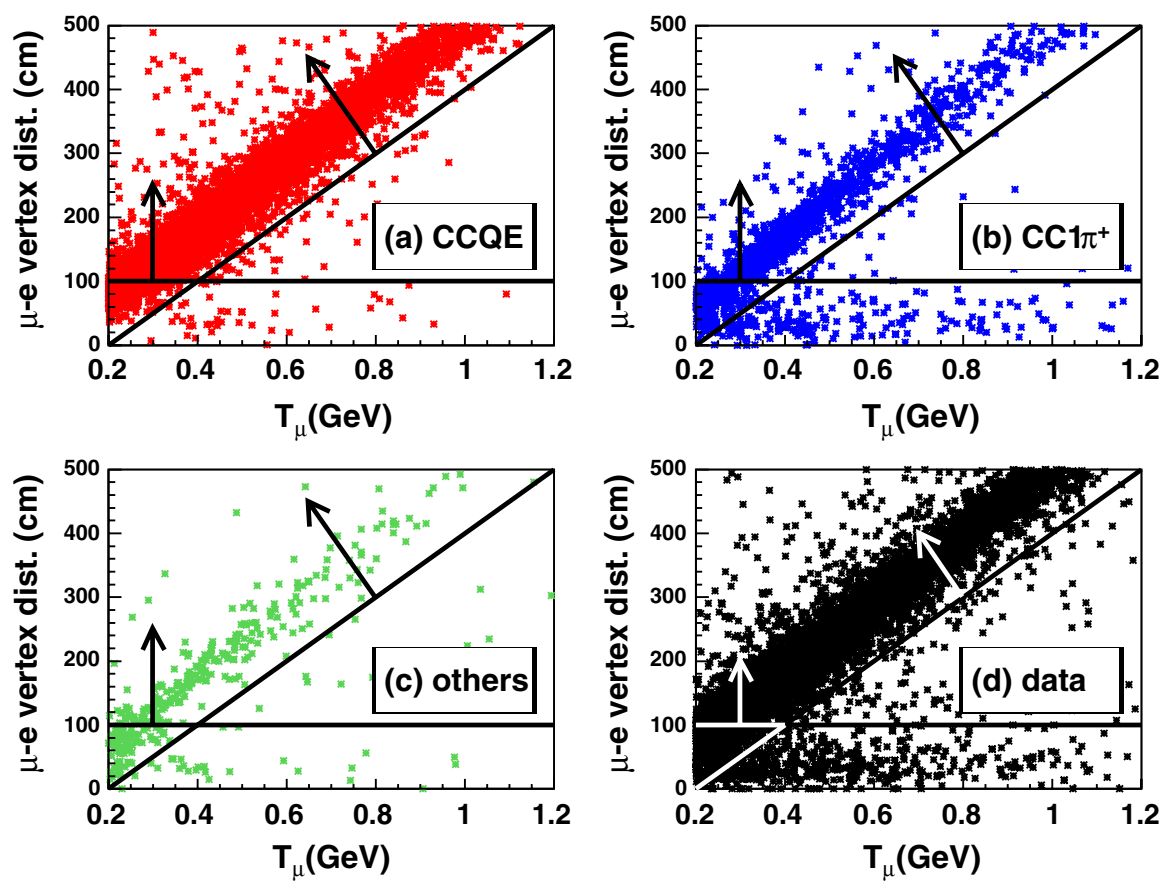

FIG. 6 (color online). Scatter plots of muon-electron vertex distance as a function of reconstructed muon kinetic energy for MC samples: (a) CCQE, (b) CC1 $\pi^{+}$, and (c) other channels. The distribution of data is shown in (d). These are events with 2-subevents and with cuts 1-6 applied. The lines with arrows indicate the region selected by the muon-electron vertex distance cut. 
the cross section calculations to correctly account for events that pass all cuts but have a true vertex with $r>$ $500 \mathrm{~cm}$. Normalizing to events with true vertex of $r<$ $500 \mathrm{~cm}$ yields an efficiency of $35 \%$.

\section{C. $\mathrm{CC} 1 \pi^{+}$background measurement}

After the selection of the $\mathrm{CCQE}$ and $\mathrm{CC} 1 \pi^{+}$candidate events (2 and 3 subevent samples, respectively), the $\mathrm{CC} 1 \pi^{+}$background to the CCQE signal is measured by adjusting the weights of the simulated $\mathrm{CC} 1 \pi^{+}$events to achieve data-MC agreement in the $Q_{\mathrm{QE}}^{2}$ distribution of the 3 subevent sample. The same weighting, applied to all simulated $\mathrm{CC} 1 \pi^{+}$events, then provides an estimate of the $\mathrm{CC} 1 \pi^{+}$background to the CCQE signal. Figure 7 shows the data and $\mathrm{MC} Q_{\mathrm{QE}}^{2}$ distributions for the two samples before the reweighting of $\mathrm{CC} 1 \pi^{+} \mathrm{MC}$ events. The 3-subevent sample is predicted to be $90 \% \mathrm{CC} 1 \pi^{+}$ and shows a large data-MC disagreement in both shape and normalization. The kinematic distribution of muons in $\mathrm{CC} 1 \pi^{+}$events is similar in both the 2 -and 3 -subevent samples as can be observed in Fig. 7. This occurs because the majority of $\mathrm{CC} 1 \pi^{+}$events that are background in the 2subevent sample are due to muon-capture or pion absorption and the reconstruction of the primary event is, to a good approximation, independent of this. In addition, the $\mu / e$ log-likelihood ratio cut (Table II and Fig. 5) is applied for both the 2- and 3-subevent samples, further ensuring that the $\mathrm{CC} 1 \pi^{+}$events are the same in both samples.

The $\mathrm{CC} 1 \pi^{+}$reweighting function [Fig. 7(b)] is a 4thorder polynomial in $Q_{\mathrm{QE}}^{2}$ and is determined from the
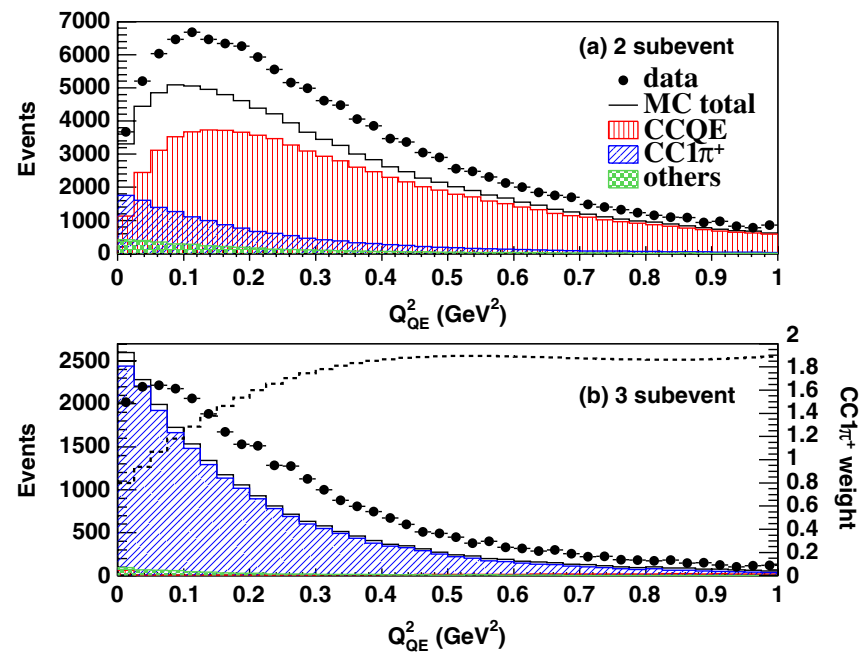

FIG. 7 (color online). Distribution of events in $Q_{\mathrm{QE}}^{2}$ for the (a) 2 and (b) 3 subevent samples before the application of the $\mathrm{CC} 1 \pi^{+}$background correction. Data and MC samples are shown along with the individual MC contributions from CCQE, $\mathrm{CC} 1 \pi^{+}$, and other channels. In (b), the dashed line shows the $\mathrm{CC} 1 \pi^{+}$reweighting function (with the $y$-axis scale on the right) as determined from the background fit procedure. ratio of data to $\mathrm{MC}$ in this sample. The 2-subevent sample shows good shape agreement between data and MC. This is because the event model for CCQE was already adjusted to match data in a previous analysis [11] that considered only the shape of the $Q_{\mathrm{QE}}^{2}$ distribution. That analysis did not consider the overall normalization of events.

In practice, this determination of the $\mathrm{CC} 1 \pi^{+}$reweighting is done iteratively as there is some CCQE background in the 3 subevent sample. An overall normalization factor is calculated for the CCQE sample to achieve data-MC agreement in the 2 subevent sample after subtraction of the $\mathrm{CC} 1 \pi^{+}$background. This is then applied to determine the CCQE background in the 3 subevent sample. The background from other channels is determined from the simulation and subtracted. This process converges after two iterations.

This method determines a correction to the $\mathrm{CC} 1 \pi^{+}$rate (as a function $Q_{\mathrm{QE}}^{2}$ ) using data from the 3-subevent sample rather than relying strictly on simulation. This reweighting is then applied to all simulated $\mathrm{CC} 1 \pi^{+}$events, in particular, those that are contained in the 2-subevent sample and form most of the background for the CCQE measurement. The error on $M_{A}^{1 \pi}$ within the resonant background model is then set to zero and the resulting error on the $\mathrm{CC} 1 \pi^{+}$ background to the $\mathrm{CCQE}$ signal from $\mathrm{CC} 1 \pi^{+}$production is determined by the coherent $\pi$-production errors and the $\pi^{+}$absorption uncertainty. The statistical errors in this procedure are negligible. Most $\mathrm{CC} 1 \pi^{+}$events that end up in the 2-subevent (CCQE) sample are due to intranuclear $\pi^{+}$absorption. This process is modeled in the event simulation as explained in Sec. IIID and is assigned a $25 \%$ uncertainty. The coherent $\pi$-production process is modeled as described in Sec. III C and is assigned a $100 \%$ uncertainty.

With the measured $\mathrm{CC} 1 \pi^{+}$background incorporated, a shape-only fit to the 2-subevent (CCQE) sample is performed to extract values for the CCQE model parameters, $M_{A}^{\text {eff }}$ and $\kappa$. This exercise is required to have a consistent description of the MiniBooNE data within the simulation after adjustment of the background. This procedure has no effect on the CCQE cross section results reported here other than very small corrections to the antineutrino background subtraction which uses these parameters. In this fit, all systematic errors and correlations are considered. The CCQE simulated sample is normalized to have the same number of events as data which is the same normalization as determined in the $\mathrm{CC} 1 \pi^{+}$background determination. The $Q_{\mathrm{QE}}^{2}$ distributions of data from the 2 and 3 subevent samples is shown together with the MC calculation in Fig. 8. The MC calculations include all the adjustments described in this section and agreement with data is good in both samples.

This shape-only fit to the 2-subevent sample yields the adjusted CCQE model parameters, $M_{A}^{\text {eff }}$ and $\kappa$, 

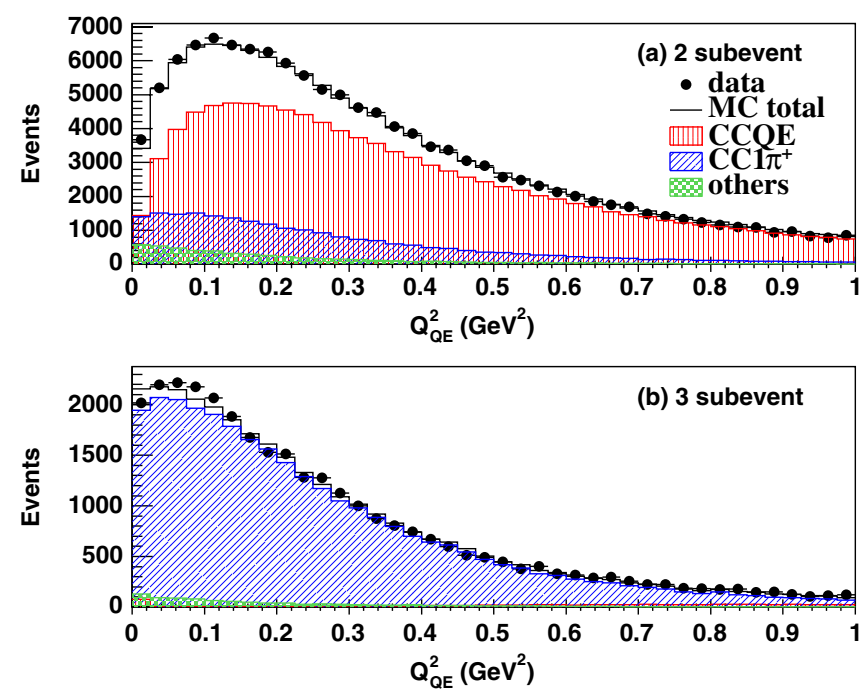

FIG. 8 (color online). Distribution of events in $Q_{\mathrm{QE}}^{2}$ for the (a) 2 and (b) 3 subevent samples after the application of the databased $\mathrm{CC} 1 \pi^{+}$background constraint and the new CCQE model parameters $M_{A}^{\text {eff }}$ and $\kappa$ as determined from the CCQE fit procedure described in the text.

$$
\begin{gathered}
M_{A}^{\mathrm{eff}}=1.35 \pm 0.17 \mathrm{GeV} / \mathrm{c}^{2} ; \quad \kappa=1.007 \pm 0.012 \\
\chi^{2} / \mathrm{dof}=47.0 / 38
\end{gathered}
$$

Figure 9 shows the $1 \sigma$ contour regions of this fit together with the results from the original MiniBooNE analysis [11]. The new fit yields different results for both $M_{A}^{\text {eff }}$ and $\kappa$ because of the improved $\mathrm{CC} 1 \pi^{+}$background estimation method used in this analysis. Note that the current

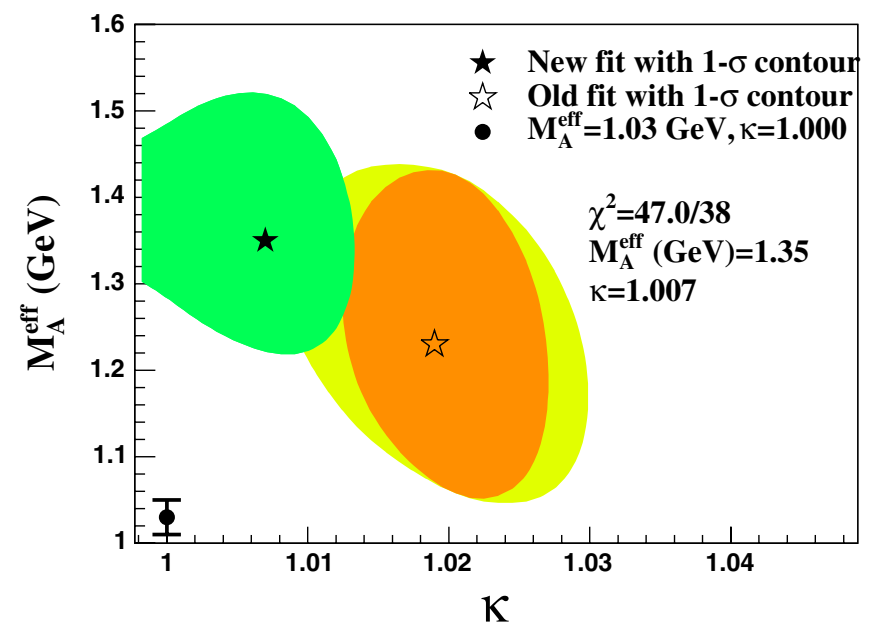

FIG. 9 (color online). The $1 \sigma$ contour plot for the $M_{A}^{\text {eff }}-\kappa$ fit. The filled star shows the best fit point and $1 \sigma$ contour extracted from this work. The open star indicates the best fit point and $1 \sigma$ contour from the previous work [11]. Two error ellipses are shown for the previous work, the larger reflects the total uncertainty ultimately included in Ref. [11]. The circle indicates the world-average value for $M_{A}$ [9]. result is consistent to within $1 \sigma$ with $\kappa=1$, unlike the previous MiniBooNE result. This fit actually provides no lower bound on $\kappa$ as the $1 \sigma$ contour is not closed for $\kappa<1$. The value for $\kappa$ is quite sensitive to the $\mathrm{CC} 1 \pi^{+}$background at the lowest $Q_{\mathrm{OE}}^{2}$ and the background in that region has decreased in this analysis. The increase in the $\mathrm{CC} 1 \pi^{+}$ background at larger $Q_{\mathrm{OE}}^{2}$ values has resulted in a larger value for the extracted $M_{A}^{\text {eff }}$. The previous and current parameter contours are consistent at the $1 \sigma$ level. Neither this nor the prior analysis result is consistent with the world-average $M_{A}$ of $1.03 \pm 0.02 \mathrm{GeV}$ [9], as can be seen in Fig. 9. The $\chi^{2} /$ dof assuming $M_{A}=1.03 \mathrm{GeV}, \kappa=$ 1 is $67.5 / 40$ corresponding to a $\chi^{2}$ probability of $\approx 0.5 \%$.

The reconstructed four-momentum transfer, $Q_{\mathrm{QE}}^{2}$, depends upon the muon energy as can be seen in Eq. (2). The reconstructed muon energy calibration has been checked by comparing the measured range of muons determined from the muon-electron vertex distance (Fig. 6) with the expected muon range determined from the energy provided by the reconstruction algorithm, which does not use this vertex distance. As an example, a comparison of the measured and expected muon ranges for muons of $400<T_{\mu}<500 \mathrm{MeV}$ is shown in Fig. 10 for both data and simulation. The agreement is good for all muon energies and verifies the energy calibration to $2 \%$, well within the errors calculated by the simulation. This also shows that any light produced by hadronic particles in the neutrino interaction (for both CCQE and background channels) is adequately simulated and considered in the reconstruction.

A final and more complete check that the simulation correctly models the data can be made by examining the 2 dimensional muon kinetic energy and angle $\left(T_{\mu}, \cos \theta_{\mu}\right)$ distributions. While Fig. 8 shows that data is welldescribed in $Q_{\mathrm{QE}}^{2}$, the adjusted model may not be adequate

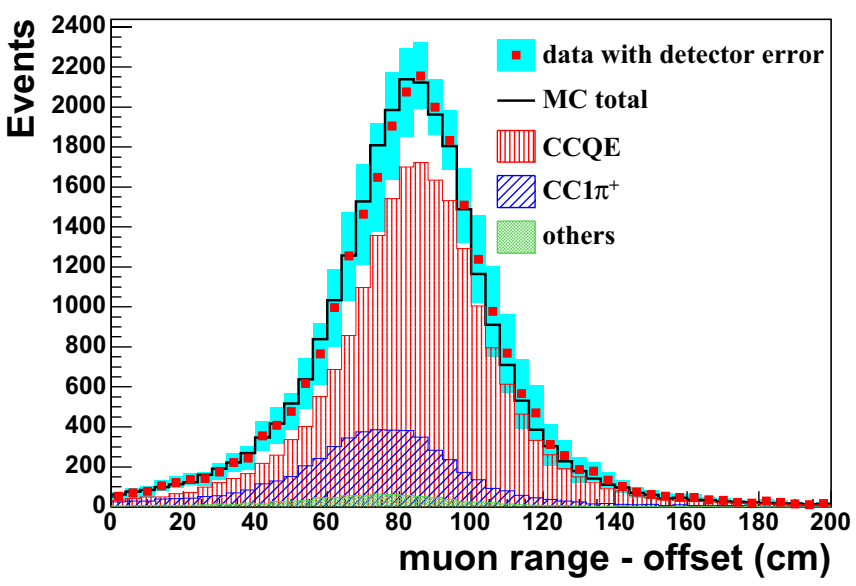

FIG. 10 (color online). Distributions, for both data and simulation, of the measured muon range for muons with $400<T_{\mu}<$ $500 \mathrm{MeV}$. The subtracted offset, $(500 \times T \mu(\mathrm{GeV})-100) \mathrm{cm}$, corresponds to the solid diagonal line in Fig. 6. 


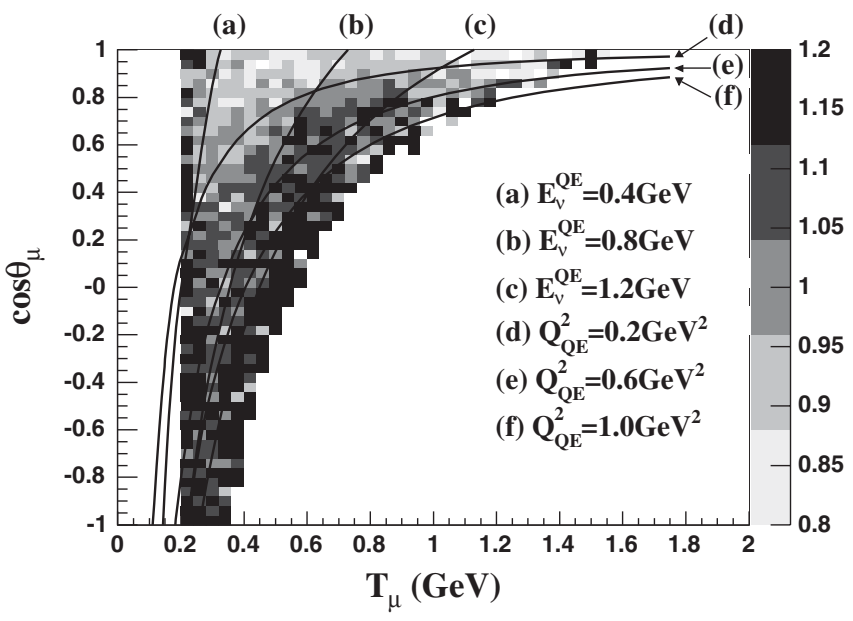

FIG. 11. Ratio of $\nu_{\mu} \mathrm{CCQE}$ data to simulation as a function of reconstructed muon kinetic energy and angle. The simulation includes adjustment of the $\mathrm{CC} 1 \pi^{+}$backgrounds based on MiniBooNE data but is that prior to any CCQE model tuning (it assumes $M_{A}=1.03 \mathrm{GeV}, \kappa=1$ ). The prediction has been normalized to the data. If the simulation modeled the data perfectly, this ratio would be unity. Contours of constant $E_{\nu}$ and $Q^{2}$ are overlaid, and only bins with $>20$ events in the data are plotted.

when applied to the $\left(T_{\mu}, \cos \theta_{\mu}\right)$ distribution of events. This could occur if an adjustment in $Q_{\mathrm{QE}}^{2}$ is hiding an incorrect neutrino energy distribution. The ratio of data to simulation in $\left(T_{\mu}, \cos \theta_{\mu}\right)$ after correction of the $\mathrm{CC} 1 \pi^{+}$ background and before the new CCQE model parameters are applied is shown in Fig. 11. The ratio after all corrections is shown in Fig. 12 and is much closer to unity throughout the muon phase space. As can be seen in

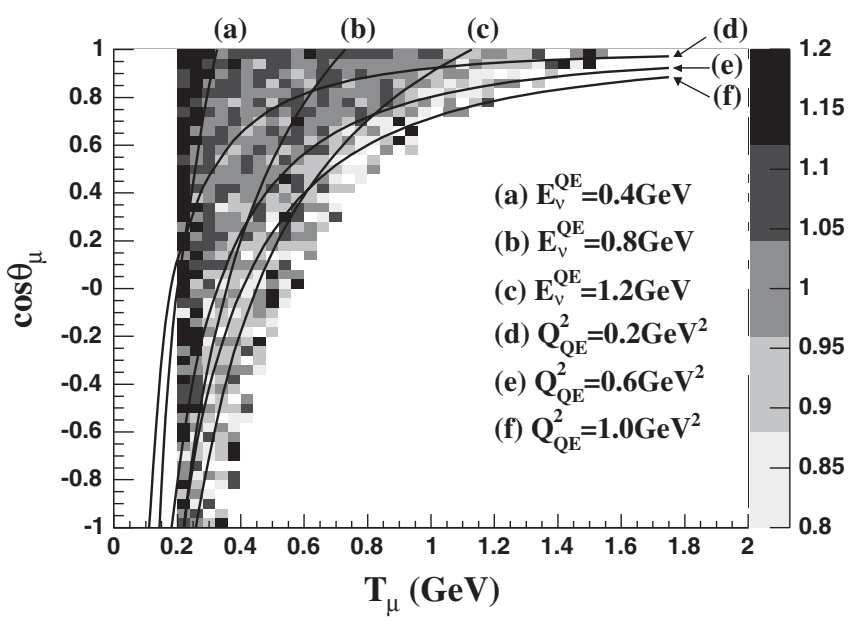

FIG. 12. Ratio of $\nu_{\mu} \mathrm{CCQE}$ data to simulation as a function of reconstructed muon kinetic energy and angle. The simulation includes the adjusted $\mathrm{CC} 1 \pi^{+}$background prediction and the new CCQE model parameters $\left(M_{A}^{\text {eff }}=1.35, \kappa=1.007\right)$ measured from MiniBooNE data. Compare to Fig. 11.
Fig. 11, the regions of constant ratio mainly follow lines of constant $Q_{\mathrm{QE}}^{2}$ and not $E_{\nu}^{\mathrm{QE}}$. Also, almost no structure remains in Fig. 12. The exception is band of $\approx 20 \%$ disagreement at $E_{\nu}^{\mathrm{QE}} \approx 0.4 \mathrm{GeV}$ where the error on the neutrino flux is of that order [Fig. 2(b).] This data-simulation agreement provides additional support for our procedure of adjusting only the $Q_{\mathrm{QE}}^{2}$ behavior of the model and not the energy distribution of the neutrino flux.

\section{Extraction of the cross sections}

With the $\mathrm{CC} 1 \pi^{+}$interaction background prediction determined from experimental data (Sec. IV C) and the remaining channels predicted from the interaction model (Sec. III), the cross section for the CCQE interaction can be extracted. A total of 146070 events pass the CCQE selection $\left(\mathrm{Sec}\right.$. IV B) resulting from $5.58 \times 10^{20}$ protons on target (POT) collected between August 2002 and December 2005. The efficiency for CCQE events passing these cuts is calculated to be $26.6 \%$ for CCQE events with true vertices within a $550 \mathrm{~cm}$ radius from the center of the detector tank. The sample is estimated to contain $23.0 \%$ background events. These numbers, together with a breakdown of predicted backgrounds, are summarized in Table III.

The background is dominated by $\mathrm{CC} 1 \pi^{+}$interactions, which are estimated to be $18.4 \%$ of the $\mathrm{CCQE}$ candidate sample. Their predicted distribution in $Q_{\mathrm{QE}}^{2}$ is shown in Fig. 8(a). As can be seen, this background is a substantial fraction of the sample in the lowest $Q_{\mathrm{QE}}^{2}$ region. The majority (52\%) of the $\mathrm{CC} 1 \pi^{+}$background is predicted to be events in which the $\pi^{+}$is absorbed in the initial target nucleus. These are defined as "CCQE-like" in that they contain a muon and no pions in the final state. The remaining $\mathrm{CC} 1 \pi^{+}$background consists of $\mathrm{CC} 1 \pi^{+}$events where the $\pi^{+}$is absorbed outside of the target nucleus (33\%), is

TABLE III. Summary of the final CCQE event sample including a breakdown of the estimated backgrounds from individual channels. The fraction is relative to the total measured sample. The channel nomenclature is defined in Table I.

\begin{tabular}{lcc}
\hline \hline $\begin{array}{l}\text { integrated protons on target } \\
\text { energy-integrated } \nu_{\mu} \text { flux }\end{array}$ & $\begin{array}{c}5.58 \times 10^{20} \\
\text { CCQE candidate events }\end{array}$ & $\begin{array}{c}2.88 \times 10^{11} \nu_{\mu} / \mathrm{cm}^{2} \\
\text { CCQE efficiency }(R<550 \mathrm{~cm})\end{array}$ \\
background channel & events & $26.6 \%$ \\
fraction \\
$\mathrm{NCE}$ & 45 & $<0.1 \%$ \\
$\mathrm{CC} 1 \pi^{+}$ & 26866 & $18.4 \%$ \\
$\mathrm{CC} 1 \pi^{0}$ & 3762 & $2.6 \%$ \\
$\mathrm{NC} 1 \pi^{ \pm}$ & 535 & $0.4 \%$ \\
$\mathrm{NC} 1 \pi^{0}$ & 43 & $<0.1 \%$ \\
other $\nu_{\mu}$ & 328 & $0.2 \%$ \\
all non- $\nu_{\mu}$ & 1977 & $1.4 \%$ \\
total background & 33556 & $23.0 \%$ \\
\hline \hline
\end{tabular}


not identified due to a missed muon decay (11\%), or undergoes charge exchange in the nucleus or detector medium (4\%), These last three classes of $\mathrm{CC} 1 \pi^{+}$backgrounds are not considered CCQE-like. All $\mathrm{CC} 1 \pi^{+}$background events, including those that are CCQE-like, are subtracted from the data to obtain the final CCQE cross section results. However, to facilitate examination of the model used for these processes, the effective cross section for CCQE-like background events is separately reported in the appendix.

In this analysis, the small contamination of $\bar{\nu}_{\mu}, \nu_{e}$, and $\bar{\nu}_{e}$ interactions are treated as background and are subtracted from the data based on their MC prediction (see Table III). The majority of these are $\bar{\nu}_{\mu}$ CCQE interactions. The same $M_{A}^{\text {eff }}$ and $\kappa$ as measured in the $\nu_{\mu}$ CCQE sample are used to predict non- $\nu_{\mu} \mathrm{CCQE}$ events. These parameters have been shown to adequately reproduce the MiniBooNE CCQE data collected in antineutrino mode [52].

To extract differential cross sections in muon kinematic variables, the reconstructed kinematics are corrected for detector-specific effects. A correction procedure is implemented using an "unfolding" process based on the detector simulation. We employ an "iterative Bayesian" method [53] to avoid the problem of amplification of statistical fluctuations common in the "inverse response matrix" method [54]. A disadvantage to the iterative Bayesian method is that the result depends on the initial CCQE model assumptions (the "prior" probability). However, this problem is addressed by an iterative method that uses the extracted signal distribution to correct the predicted distributions and repeating this procedure. In practice, the simulation was already tuned to reproduce the data based on previous work [11], and the result from the first iteration shows satisfactory convergence. The systematic uncertainty in this procedure is determined from the difference between the initial (0th) and final (1st) iterations of the algorithm and by examining the dependence of the final values on the initial model assumptions.

The various correction and normalization factors can then be brought together in a single expression used to extract the CCQE cross section for the $i$ th bin of a particular kinematic variable,

$$
\sigma_{i}=\frac{\sum_{j} U_{i j}\left(d_{j}-b_{j}\right)}{\epsilon_{i} \cdot T \cdot \Phi}
$$

where the index $j$ labels the reconstructed bin and $i$ labels the unfolded (estimate of the true) bin. In this equation, $U_{i j}$ is the unfolding matrix, $\epsilon_{i}$ is the efficiency, $T$ is the number of neutrons in the fiducial volume, and $\Phi$ is the neutrino flux. This expression is used to obtain the double and single differential cross sections, $\frac{d^{2} \sigma}{d T_{\mu} d \cos \theta_{\mu}}$, and $\frac{d \sigma}{d Q_{\mathrm{QE}}^{2}}$, respectively, after the multiplication of the appropriate bin width factors. Note that the choice of normalization yields cross sections "per neutron". Here, the flux, $\Phi$, is a single number $\left(2.88 \times 10^{11} \nu_{\mu} / \mathrm{cm}^{2}\right)$ and is determined by integrating the BNB flux over $0<E_{\nu}<3 \mathrm{GeV}$. Therefore, these differential cross sections are "flux-integrated."

An additional quantity, the flux-unfolded CCQE cross section as a function of neutrino energy, $\sigma\left[E_{\nu}^{\mathrm{QE}, \mathrm{RFG}}\right]$, is extracted from this same expression with the replacement of the total flux, $\Phi$, with the flux in a particular neutrino energy bin, $\Phi_{i}$. The unfolding procedure is used to correct the data from bins of reconstructed neutrino energy, $E_{\nu}^{\mathrm{QE}}$, [using Eq. (1)] to an estimate of the true neutrino energy, $E_{\nu}^{\mathrm{QE}, \mathrm{RFG}}$. It is important to note that, unlike the differential cross sections, $\frac{d^{2} \sigma}{d T_{\mu} d \cos \theta_{\mu}}$ and $\frac{d \sigma}{d Q_{\mathrm{QE}}^{2}}$, the calculation of this cross section relies on the interaction model to connect $E_{\nu}^{\mathrm{QE}}$ to $E_{\nu}^{\mathrm{QE}, \mathrm{RFG}}$. The superscript " $\mathrm{RFG}$ " indicates the interaction model assumed in the unfolding process [8]. This procedure introduces a model dependence into this cross section, however, this method is consistent with that commonly used by experiments reporting a CCQE cross section as a function of neutrino energy. This model dependence should be considered when comparing measurements of this quantity from different experiments.

\section{E. Error analysis}

The errors on the measured cross sections result from uncertainties in the neutrino flux, background estimates, detector response, and unfolding procedure. To propagate these error sources, a "multisim" method [55] is used to calculate the errors on the final quantities by varying parameters in separate simulations. This method produces an error matrix $V_{i j}$ for each reported distribution that can then be used to extract the error on each bin $\left(\sigma_{i}=\sqrt{V_{i i}}\right)$ and the correlations between quantities of different bins.

The error matrix is calculated by generating a large number of simulated data sets with different parameter excursions, based on the estimated $1 \sigma$ uncertainties in those parameters and the correlations between them. The error matrix for a particular distribution is then calculated from these $M$ data sets,

$$
V_{i j}=\frac{1}{M} \sum_{s=1}^{M}\left(Q_{i}^{s}-\hat{Q}_{i}\right)\left(Q_{j}^{s}-\hat{Q}_{j}\right)
$$

Here, $Q_{i}^{s}$ is the quantity of interest in the $i$ th bin from the $s$ th simulation data set and $\hat{Q}_{i}$ is the "best" estimate of the parameters. The quantities of interest $Q_{i}$ could be, for instance, the number of events in each bin of $Q_{\mathrm{QE}}^{2}$ or the calculated cross section in each kinematic bin [Eq. (3)].

In practice, the errors are classified into four major contributions: the neutrino flux, background cross sections, detector modeling, and unfolding procedure. The parameters within each of these groups are varied independently so the resultant error matrices from the individual groups 
can be added to form the total error matrix. For the neutrino flux and background cross section uncertainties, a reweighting method is employed which removes the difficulty of requiring hundreds of simulations with adequate statistics. In this method, each neutrino interaction event is given a new weight calculated with a particular parameter excursion. This is performed considering correlations between parameters and allows each generated event to be reused many times saving significant CPU time. The nature of the detector uncertainties does not allow for this method of error evaluation as parameter uncertainties can only be applied as each particle or optical photon propagates through the detector. Approximately 100 different simulated data sets are generated with the detector parameters varied according to the estimated $1 \sigma$ errors including correlations. Equation (4) is then used to calculate the detector error matrix. The error on the unfolding procedure is calculated from the difference in final results when using different input model assumptions (Sec. IV D). The statistical error on data is not added explicitly but is included via the statistical fluctuations of the simulated data sets (which have the same number of events as the data).

The final uncertainties are reported in the following sections. The breakdown among the various contributions are summarized and discussed in Sec. V D. For simplicity, the full error matrices are not reported for all distributions. Instead, the errors are separated into a total normalization error, which is an error on the overall scale of the cross section, and a "shape error" which contains the uncertainty that does not factor out into a scale error. This allows for a distribution of data to be used (e.g. in a model fit) with an overall scale error for uncertainties that are completely correlated between bins, together with the remaining bindependent shape error.

\section{RESULTS AND DISCUSSION}

\section{A. CCQE flux-integrated double differential cross section}

The flux-integrated, double differential cross section per neutron, $\frac{d^{2} \sigma}{d T_{\mu} d \cos \theta_{\mu}}$, for the $\nu_{\mu}$ CCQE process is extracted as described in Sec. IVD and is shown in Fig. 13 for the kinematic range, $-1<\cos \theta_{\mu}<+1,0.2<T_{\mu}(\mathrm{GeV})<$ 2.0. The errors, for $T_{\mu}$ outside of this range, are too large to allow a measurement. Also, bins with low event population near or outside of the kinematic edge of the distribution (corresponding to large $E_{\nu}$ ) do not allow for a measurement and are shown as zero in the plot. The numerical values for this double differential cross section are provided in Table VI in the appendix.

The flux-integrated CCQE total cross section, obtained by integrating the double differential cross section (over $-1<\cos \theta_{\mu}<+1,0<T_{\mu}(\mathrm{GeV})<\infty$ ), is measured to be $9.429 \times 10^{-39} \mathrm{~cm}^{2}$. The total normalization error on this measurement is $10.7 \%$.

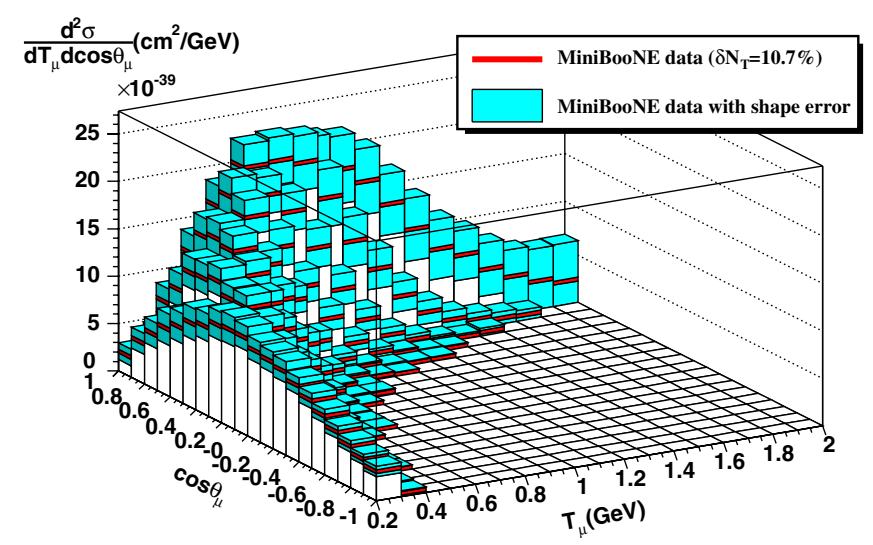

FIG. 13 (color online). Flux-integrated double differential cross section per target neutron for the $\nu_{\mu}$ CCQE process. The dark bars indicate the measured values and the surrounding lighter bands show the shape error. The overall normalization (scale) error is $10.7 \%$. Numerical values are provided in Table VI in the Appendix.

The kinematic quantities, $T_{\mu}$ and $\cos \theta_{\mu}$, have been corrected for detector resolution effects only (Sec. IV D). Thus, this result is the most model-independent measurement of this process possible with the MiniBooNE detector. No requirements on the nucleonic final state are used to define this process. The neutrino flux is an absolute prediction [19] and has not been adjusted based on measured processes in the MiniBooNE detector.

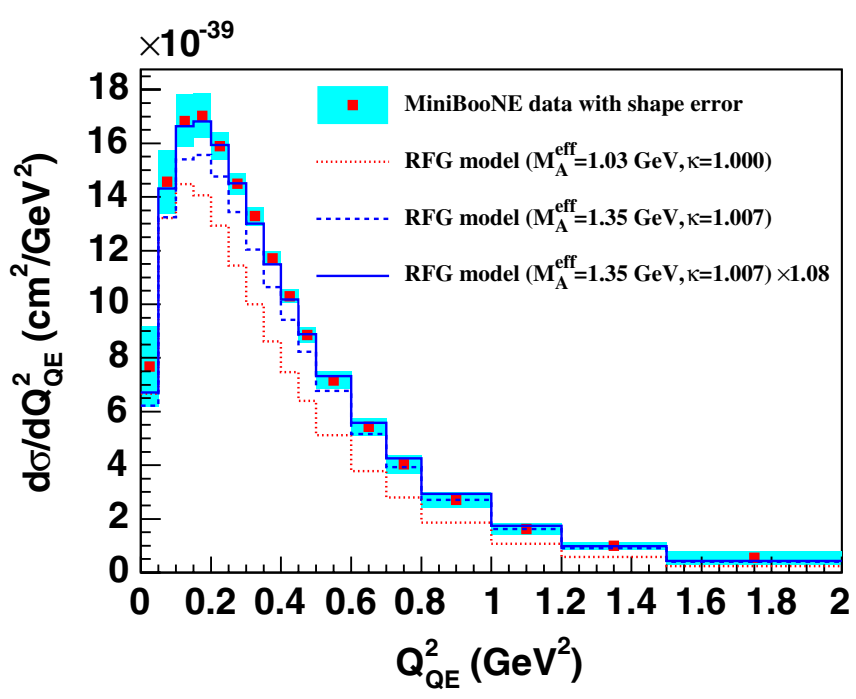

FIG. 14 (color online). Flux-integrated single differential cross section per target neutron for the $\nu_{\mu}$ CCQE process. The measured values are shown as points with the shape error as shaded bars. Calculations from the NUANCE RFG model with different assumptions for the model parameters are shown as histograms. Numerical values are provided in Table IX in the appendix. 


\section{B. Flux-integrated single differential cross section}

The flux-integrated, single differential cross section per neutron, $\frac{d \sigma}{d Q_{\mathrm{QE}}^{2}}$, has also been measured and is shown in Fig. 14. The quantity $Q_{\mathrm{QE}}^{2}$ is defined in Eq. (2) and depends only on the (unfolded) quantities $T_{\mu}$ and $\cos \theta_{\mu}$. It should be noted that the efficiency for events with $T_{\mu}<200 \mathrm{MeV}$ is not zero because of difference between reconstructed and unfolded $T_{\mu}$. The calculation of efficiency for these (low- $Q_{\mathrm{QE}}^{2}$ ) events depends only on the model of the detector response, not on an interaction model and the associated uncertainty is propagated to the reported results.

In addition to the experimental result, Fig. 14 also shows the prediction for the CCQE process from the NUANCE simulation with three different sets of parameters in the underlying RFG model. The predictions are absolutely normalized and have been integrated over the MiniBooNE flux. The RFG model is plotted assuming both the world-averaged CCQE parameters $\left(M_{A}=\right.$ $1.03 \mathrm{GeV}, \kappa=1.000$ ) [9] and the CCQE parameters extracted from this analysis $\left(M_{A}=1.35 \mathrm{GeV}, \kappa=1.007\right)$ in a shape-only fit. The model using the world-averaged CCQE parameters underpredicts the measured differential cross section values by $20 \%-30 \%$, while the model using the CCQE parameters extracted from this shape analysis are within $\approx 8 \%$ of the data, consistent within the normalization error $(\approx 10 \%)$. To further illustrate this, the model calculation with the CCQE parameters from this analysis scaled by 1.08 is also plotted and shown to be in good agreement with the data.

\section{Flux-unfolded CCQE cross section as a function of neutrino energy}

The flux-unfolded CCQE cross section per neutron $\sigma\left[E_{\nu}^{\mathrm{QE}, \mathrm{RFG}}\right]$, as a function of the true neutrino energy $E_{\nu}^{\mathrm{QE}, \mathrm{RFG}}$, is shown in Fig. 15. These numerical values are tabulated in Table $\mathrm{X}$ in the appendix. The quantity $E_{\nu}^{\mathrm{QE}, \mathrm{RFG}}$ is a (model-dependent) estimate of the neutrino energy obtained after correcting for both detector and nuclear model resolution effects. These results depend on the details of the nuclear model used for the calculation. The dependence is only weak in the peak of the flux distribution but becomes strong for $E_{\nu}<0.5 \mathrm{GeV}$ and $E_{\nu}>1.2 \mathrm{GeV}$, i.e., in the "tails" of the flux distribution.

In Fig. 15, the data are compared with the NUANCE implementation of the RFG model with the world average parameter values, $\left(M_{A}^{\text {eff }}=1.03 \mathrm{GeV}, \kappa=1.000\right)$ and with the parameters extracted from this work $\left(M_{A}^{\text {eff }}=\right.$ $1.35 \mathrm{GeV}, \kappa=1.007)$. These are absolute predictions from the model (not scaled or renormalized). At the average energy of the MiniBooNE flux $(\approx 800 \mathrm{MeV})$, the extracted cross section is $\approx 30 \%$ larger than the RFG model prediction with world average parameter values. The RFG model, with parameter values extracted from
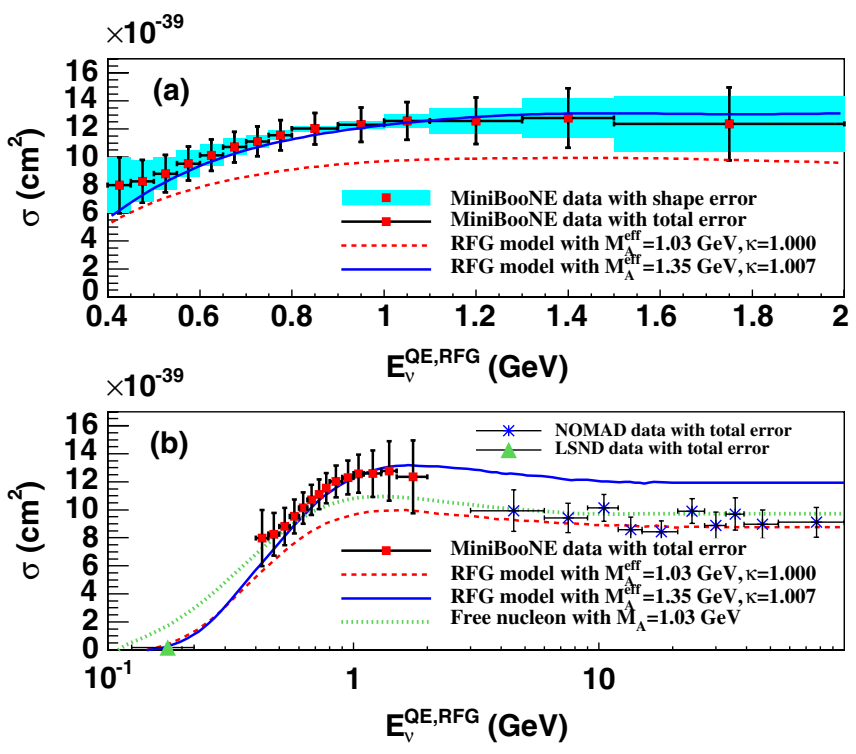

FIG. 15 (color online). Flux-unfolded MiniBooNE $\nu_{\mu}$ CCQE cross section per neutron as a function of neutrino energy. In (a), shape errors are shown as shaded boxes along with the total errors as bars. In (b), a larger energy range is shown along with results from the LSND [56] and NOMAD [10] experiments. Also shown are predictions from the NUANCE simulation for an RFG model with two different parameter variations and for scattering from free nucleons with the world-average $M_{A}$ value. Numerical values are provided in Table $\mathrm{X}$ in the appendix.

the shape-only fit to this data better reproduces the data over the entire measured energy range.

Figure 15(b) shows these CCQE results together with those from the LSND [56] and NOMAD [10] experiments. It is interesting to note that the NOMAD results are better described with the world average $M_{A}^{\text {eff }}$ and $\kappa$ values. Also shown for comparison in Fig. 15(b) is the predicted cross section assuming the CCQE interaction occurs on free nucleons with the world average $M_{A}$ value. The cross sections reported here exceed the free nucleon value for $E_{\nu}$ above $0.7 \mathrm{GeV}$.

\section{Error summary}

As described in Sec. IV E, (correlated) systematic and statistical errors are propagated to the final results. These

TABLE IV. Contribution to the total normalization uncertainty from each of the various systematic error categories.

\begin{tabular}{lc}
\hline \hline source & normalization error $(\%)$ \\
\hline neutrino flux prediction & 8.66 \\
background cross sections & 4.32 \\
detector model & 4.60 \\
kinematic unfolding procedure & 0.60 \\
statistics & 0.26 \\
total & 10.7 \\
\hline \hline
\end{tabular}




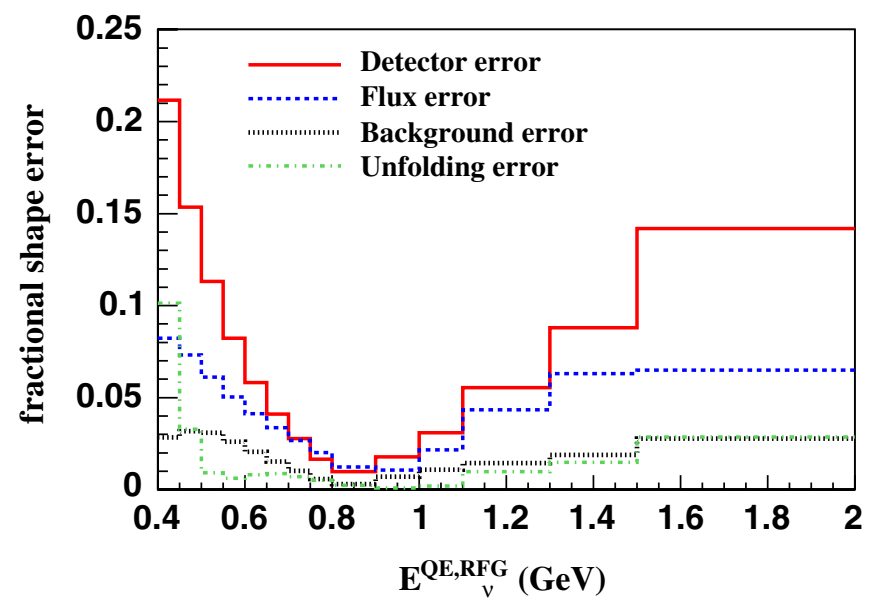

FIG. 16 (color online). Fractional shape error on the MiniBooNE $\nu_{\mu}$ CCQE flux-unfolded cross section separated into major components. The overall normalization error of $10.7 \%$ is not shown.

errors are separated into normalization and shape uncertainties. The contributions from each error source on the total normalization uncertainty are summarized in Table IV. As is evident, the neutrino flux uncertainty dominates the overall normalization error on the extracted CCQE cross sections. However, the uncertainty on the flux prediction is a smaller contribution to the shape error on the cross sections. This can be seen in Fig. 16 which shows the contribution from the four major sources to the shape error on the total (flux-unfolded) cross section.

The detector model uncertainty dominates the shape error, especially at low and high energies. This is because errors in the detector response (mainly via uncertainties in visible photon processes) will result in errors on the reconstructed energy. These errors grow in the tails of the neutrino flux distribution due to feed-down from events in the flux peak. This type of measurement usually has large errors due to non-negligible uncertainties in the $\mathrm{CC} 1 \pi^{+}$background predictions. In this measurement, that error is reduced through direct measurement of the $\mathrm{CC} 1 \pi^{+}$background. However, this error is not completely eliminated due to the residual uncertainty on the rate of intranuclear pion absorption that is included. This uncertainty is not as important for the measurement of the CCQE cross section measurement as a function of energy but is a large contribution to the error at low $Q_{\mathrm{QE}}^{2}$ in the differential distributions.

The unfolding error is small in the region of the flux peak but grows in the high- and low-energy region because of the uncertainty in the feed-down from other energy bins, similar to that described for the detector model.

\section{CONCLUSIONS}

In this work, we report measurements of absolute cross sections for the CCQE interaction using high-statistics samples of $\nu_{\mu}$ interactions on carbon. These include the first measurement of the double differential cross section, $\frac{d^{2} \sigma}{d T_{\mu} d \cos \theta_{\mu}}$, measurement of the single differential cross section, $\frac{d \sigma}{d Q_{\mathrm{QE}}^{2}}$, and the flux-unfolded CCQE cross section, $\sigma\left[E_{\nu}^{\mathrm{QE}, \mathrm{RFG}}\right]$. The double differential cross section contains the most complete and model-independent information that is available from MiniBooNE on the CCQE process. It is the main result from this work and should be used as the preferred choice for comparison to theoretical models of CCQE interactions on nuclear targets.

The reported cross section is significantly larger ( $\approx 30 \%$ at the flux average energy) than what is commonly assumed for this process assuming a relativistic Fermi Gas model (RFG) and the world-average value for the axial mass, $M_{A}=1.03 \mathrm{GeV}$ [9]. In addition, the $Q_{\mathrm{QE}}^{2}$ distribution of this data shows a significant excess of events over this expectation at higher $Q_{\mathrm{QE}}^{2}$ even if the data is normalized to the prediction over all $Q_{\mathrm{QE}}^{2}$. This leads to an extracted axial mass from a "shape-only" fit of the $Q_{\mathrm{QE}}^{2}$ distribution of $M_{A}^{\text {eff }}=1.35 \pm 0.17 \mathrm{GeV}$, significantly higher than the historical world-average value.

These two observations, unexpectedly large values for the extracted cross section and $M_{A}^{\text {eff }}$, are experimentally separate. However, within the model prediction, a larger value for $M_{A}$ implies a larger cross section because the CCQE cross section increases approximately linearly with $M_{A}$. The predicted CCQE cross section with this higher value of $M_{A}$ agrees with the measurement within the normalization error of the experiment $(\approx 10 \%)$. While this may be simply a coincidence, it is important to note.

In recent years, there has been significant effort to improve the theoretical description of the CCQE interaction on nuclear targets $[16,17]$; however, there seems to be no simple explanation for both the higher cross section and the harder $Q_{\mathrm{QE}}^{2}$ distribution of events (resulting in a larger $M_{A}^{\text {eff }}$ ) as evidenced by the MiniBooNE CCQE data. Nuclear effects can have some impact on the measured $M_{A}^{\text {eff }}$, but it is not obvious that they are large enough. Also, it is expected that such effects should reduce the cross section, not increase it. This can be seen in Fig. 15 where the cross section for the CCQE interaction on free nucleons is compared to that from bound nucleons (in an RFG model). Note the reduction in cross section from free to bound nucleons. It is interesting that the MiniBooNE measurement is also larger than this free nucleon value (at least at higher energies). This may indicate a significant contribution from neglected mechanisms for CCQE-like scattering from a nucleus such as multinucleon processes (for example, Ref. [17]). This may explain both the higher cross section and the harder $Q^{2}$ spectrum, but has not yet been explicitly tested. It may also be relevant for the difference between these results and those of NOMAD (or other experiments) where the observation of recoil nucleons enter the definition of a CCQE event. An important test 
for such models will be their ability to accurately reproduce the MiniBooNE double differential cross sections at least as well as the RFG model assuming a higher axial mass value.

As yet, there is no easily recognized solution to explain the difference between the CCQE cross sections measured in MiniBooNE at lower neutrino energy $\left(E_{\nu}<2 \mathrm{GeV}\right)$ and the NOMAD results at higher neutrino energies $\left(E_{\nu}>\right.$ $3 \mathrm{GeV}$ ). Model-independent measurements of the CCQE cross section anticipated from SciBooNE [57], MicroBooNE [58], and MINERvA [59] as well as the T2K [6] and NOvA [5] near detectors running with $2<$ $E_{\nu}<20 \mathrm{GeV}$, will be important to help resolve these results.

\section{ACKNOWLEDGMENTS}

This work was conducted with support from Fermilab, the U.S. Department of Energy, and the National Science Foundation .

\section{APPENDIX A: TABULATION OF RESULTS}

This appendix contains tables of numerical values corresponding to various plots appearing in the main body of the paper. In addition, the effective cross section for the CCQE-like background to the CCQE measurement is tabulated. These tables are also available via the MiniBooNE website [60].

\section{Predicted $\nu_{\mu}$ flux}

Table V lists the predicted $\nu_{\mu}$ flux (Fig. 2) at the MiniBooNE detector in $50 \mathrm{MeV}$-wide neutrino energy bins. The flux is normalized to protons on target (POT). The mean energy is $788 \mathrm{MeV}$ and the integrated flux over the energy range $\left(0.0<E_{\nu}<3.0 \mathrm{GeV}\right)$ is $5.16 \times$ $10^{-10} \nu_{\mu} / \mathrm{POT} / \mathrm{cm}^{2}$. For this analysis, the total POT collected is $5.58 \times 10^{20}$ yielding an integrated flux of $2.88 \times$ $10^{11} \nu_{\mu} / \mathrm{cm}^{2}$.

\section{CCQE flux-integrated double differential cross section}

Table VI contains the flux-integrated $\nu_{\mu} \mathrm{CCQE}$ double differential cross section values $\left(\frac{d^{2} \sigma}{d T_{\mu} d \cos \theta_{\mu}}\right)$ in bins of muon energy $T_{\mu}$ and cosine of the muon scattering angle with respect to the incoming neutrino direction (in the lab frame) $\cos \theta_{\mu}$. These values correspond to the plot of Fig. 13. The integrated value over the region $(-1<$ $\cos \theta_{\mu}<+1$ and $\left.0<T_{\mu}<\infty\right)$ is $9.429 \times 10^{-39} \mathrm{~cm}^{2}$. The total normalization uncertainty is $10.7 \%$. Table VII present an analogous summary of the shape error for each bin.

\section{CCQE-like backgrounds}

As explained in Sec. IV D, the $\mathrm{CC} 1 \pi^{+}$interaction with intranuclear pion absorption forms a "CCQE-like" background in that the final state is indistinguishable from the

TABLE V. Predicted $\nu_{\mu}$ flux at the MiniBooNE detector.

\begin{tabular}{cccccc}
\hline \hline $\begin{array}{c}E_{\nu} \text { bin } \\
(\mathrm{GeV})\end{array}$ & $\begin{array}{c}\nu_{\mu} \text { flux } \\
\left(\nu_{\mu} / \mathrm{POT} / \mathrm{GeV} / \mathrm{cm}^{2}\right)\end{array}$ & $\begin{array}{c}E_{\nu} \text { bin } \\
(\mathrm{GeV})\end{array}$ & $\begin{array}{c}\nu_{\mu} \text { flux } \\
\left(\nu_{\mu} / \mathrm{POT} / \mathrm{GeV} / \mathrm{cm}^{2}\right)\end{array}$ & $\begin{array}{c}E_{\nu} \text { bin } \\
(\mathrm{GeV})\end{array}$ & $\begin{array}{c}\nu_{\mu} \text { flux } \\
\left(\nu_{\mu} / \mathrm{POT} / \mathrm{GeV} / \mathrm{cm}^{2}\right)\end{array}$ \\
\hline $0.00-0.05$ & $4.54 \times 10^{-11}$ & $1.00-1.05$ & $3.35 \times 10^{-10}$ & $2.00-2.05$ & $1.92 \times 10^{-11}$ \\
$0.05-0.10$ & $1.71 \times 10^{-10}$ & $1.05-1.10$ & $3.12 \times 10^{-10}$ & $2.05-2.10$ & $1.63 \times 10^{-11}$ \\
$0.10-0.15$ & $2.22 \times 10^{-10}$ & $1.10-1.15$ & $2.88 \times 10^{-10}$ & $2.10-2.15$ & $1.39 \times 10^{-11}$ \\
$0.15-0.20$ & $2.67 \times 10^{-10}$ & $1.15-1.20$ & $2.64 \times 10^{-10}$ & $2.15-2.20$ & $1.19 \times 10^{-11}$ \\
$0.20-0.25$ & $3.32 \times 10^{-10}$ & $1.20-1.25$ & $2.39 \times 10^{-10}$ & $2.20-2.25$ & $1.03 \times 10^{-11}$ \\
$0.25-0.30$ & $3.64 \times 10^{-10}$ & $1.25-1.30$ & $2.14 \times 10^{-10}$ & $2.25-2.30$ & $8.96 \times 10^{-12}$ \\
$0.30-0.35$ & $3.89 \times 10^{-10}$ & $1.30-1.35$ & $1.90 \times 10^{-10}$ & $2.30-2.35$ & $7.87 \times 10^{-12}$ \\
$0.35-0.40$ & $4.09 \times 10^{-10}$ & $1.35-1.40$ & $1.67 \times 10^{-10}$ & $2.35-2.40$ & $7.00 \times 10^{-12}$ \\
$0.40-0.45$ & $4.32 \times 10^{-10}$ & $1.40-1.45$ & $1.46 \times 10^{-10}$ & $2.40-2.45$ & $6.30 \times 10^{-12}$ \\
$0.45-0.50$ & $4.48 \times 10^{-10}$ & $1.45-1.50$ & $1.26 \times 10^{-10}$ & $2.45-2.50$ & $5.73 \times 10^{-12}$ \\
$0.50-0.55$ & $4.56 \times 10^{-10}$ & $1.50-1.55$ & $1.08 \times 10^{-10}$ & $2.50-2.55$ & $5.23 \times 10^{-12}$ \\
$0.55-0.60$ & $4.58 \times 10^{-10}$ & $1.55-1.60$ & $9.20 \times 10^{-11}$ & $2.55-2.60$ & $4.82 \times 10^{-12}$ \\
$0.60-0.65$ & $4.55 \times 10^{-10}$ & $1.60-1.65$ & $7.80 \times 10^{-11}$ & $2.60-2.65$ & $4.55 \times 10^{-12}$ \\
$0.65-0.70$ & $4.51 \times 10^{-10}$ & $1.65-1.70$ & $6.57 \times 10^{-11}$ & $2.65-2.70$ & $4.22 \times 10^{-12}$ \\
$0.70-0.75$ & $4.43 \times 10^{-10}$ & $1.70-1.75$ & $5.52 \times 10^{-11}$ & $2.70-2.75$ & $3.99 \times 10^{-12}$ \\
$0.75-0.80$ & $4.31 \times 10^{-10}$ & $1.75-1.80$ & $4.62 \times 10^{-11}$ & $2.75-2.80$ & $3.84 \times 10^{-12}$ \\
$0.80-0.85$ & $4.16 \times 10^{-10}$ & $1.80-1.85$ & $3.86 \times 10^{-11}$ & $2.80-2.85$ & $3.63 \times 10^{-12}$ \\
$0.85-0.90$ & $3.98 \times 10^{-10}$ & $1.85-1.90$ & $3.23 \times 10^{-11}$ & $2.85-2.90$ & $3.45 \times 10^{-12}$ \\
$0.90-0.95$ & $3.79 \times 10^{-10}$ & $1.90-1.95$ & $2.71 \times 10^{-11}$ & $2.90-2.95$ & $3.33 \times 10^{-12}$ \\
$0.95-1.00$ & $3.58 \times 10^{-10}$ & $1.95-2.00$ & $2.28 \times 10^{-11}$ & $2.95-3.00$ & $3.20 \times 10^{-12}$ \\
\hline \hline
\end{tabular}


TABLE VI. The MiniBooNE $\nu_{\mu}$ CCQE flux-integrated double differential cross section in units of $10^{-41} \mathrm{~cm}^{2} / \mathrm{GeV}$ in $0.1 \mathrm{GeV}$ bins of $T_{\mu}$ (columns) and 0.1 bins of $\cos \theta_{\mu}$ (rows).

\begin{tabular}{|c|c|c|c|c|c|c|c|c|c|c|c|c|c|c|c|c|c|c|}
\hline $\cos \theta_{\mu} T_{\mu}(\mathrm{GeV})$ & $.2,0.3$ & $0.3,0.4$ & $0.4,0.5$ & $0.5,0.6$ & $0.6,0.7$ & $0.7,0.8$ & $0.8,0.9$ & $0.9,1.0$ & $1.0,1.1$ & $1.1,1.2$ & $1.2,1.3$ & $1.3,1.4$ & $1.4,1.5$ & $1.5,1.6$ & $1.6,1.7$ & $1.7,1.8$ & $1.8,1.9$ & $1.9,2.0$ \\
\hline$+0.9,+1.0$ & 190.0 & 326.5 & 539.2 & 901.8 & 1288 & 1633 & 1857 & 1874 & 1803 & 1636 & 1354 & 1047 & 794.0 & 687.9 & 494.3 & 372.5 & 278.3 & 227.4 \\
\hline$+0.8,+0.9$ & 401.9 & 780.6 & 1258 & 1714 & 2084 & 2100 & 2035 & 1620 & 1118 & 783.6 & 451.9 & 239.4 & 116.4 & 73.07 & 41.67 & 36.55 & $\cdots$ & $\ldots$ \\
\hline$+0.7,+0.8$ & 553.6 & 981.1 & 1501 & 1884 & 1847 & 1629 & 1203 & 723.8 & 359.8 & 156.2 & 66.90 & 26.87 & 1.527 & 19.50 & $\ldots$ & $\cdots$ & $\cdots$ & $\ldots$ \\
\hline$+0.6,+0.7$ & 681.9 & 1222 & 1546 & 1738 & 1365 & 909.6 & 526.7 & 222.8 & 81.65 & 35.61 & 11.36 & 0.131 & $\cdots$ & $\cdots$ & $\cdots$ & $\cdots$ & $\cdots$ & $\cdots$ \\
\hline$+0.5,+0.6$ & 765.6 & 1233 & 1495 & 1289 & 872.2 & 392.3 & 157.5 & 49.23 & 9.241 & 1.229 & 4.162 & $\cdots$ & $\cdots$ & $\cdots$ & $\cdots$ & $\cdots$ & $\cdots$ & $\cdots$ \\
\hline$+0.4,+0.5$ & 871.9 & 1279 & 1301 & 989.9 & 469.1 & 147.4 & 45.02 & 12.44 & 1.012 & $\ldots$ & $\cdots$ & $\ldots$ & $\ldots$ & $\ldots$ & $\ldots$ & $\ldots$ & $\ldots$ & $\ldots$ \\
\hline$+0.3,+0.4$ & 910.2 & 1157 & 1054 & 628.8 & 231.0 & 57.95 & 10.69 & $\cdots$ & $\cdots$ & $\cdots$ & $\cdots$ & $\cdots$ & $\cdots$ & $\cdots$ & $\cdots$ & $\cdots$ & $\cdots$ & $\cdots$ \\
\hline$+0.2,+0.3$ & 992.3 & 1148 & 850.0 & 394.4 & 105.0 & 16.96 & 10.93 & & & & $\cdots$ & $\cdots$ & $\cdots$ & $\cdots$ & $\cdots$ & $\cdots$ & $\cdots$ & $\cdots$ \\
\hline$+0.1,+0.2$ & 1007 & 970.2 & 547.9 & 201.5 & 36.51 & 0.844 & $\cdots$ & $\cdots$ & $\ldots$ & $\cdots$ & $\cdots$ & $\cdots$ & $\cdots$ & $\cdots$ & $\ldots$ & $\cdots$ & $\ldots$ & $\cdots$ \\
\hline $0.0,+0.1$ & 1003 & 813.1 & 404.9 & 92.93 & 11.63 & $\ldots$ & $\cdots$ & $\ldots$ & $\ldots$ & $\ldots$ & $\cdots$ & $\cdots$ & $\cdots$ & $\ldots$ & $\ldots$ & $\cdots$ & $\cdots$ & $\cdots$ \\
\hline$-0.1,0.0$ & 919.3 & 686.6 & 272.3 & 40.63 & 2.176 & $\cdots$ & $\cdots$ & $\cdots$ & $\cdots$ & $\cdots$ & $\cdots$ & $\cdots$ & $\cdots$ & $\cdots$ & $\cdots$ & $\cdots$ & $\cdots$ & $\cdots$ \\
\hline$-0.2,-0.1$ & 891.8 & 503.3 & 134.7 & 10.92 & 0.071 & $\cdots$ & $\cdots$ & $\cdots$ & $\ldots$ & $\cdots$ & $\cdots$ & $\cdots$ & $\cdots$ & $\ldots$ & $\ldots$ & $\cdots$ & $\ldots$ & $\cdots$ \\
\hline$-0.3,-0.2$ & 857.5 & 401.6 & 79.10 & 1.947 & $\ldots$ & $\ldots$ & $\ldots$ & $\ldots$ & $\ldots$ & $\ldots$ & $\ldots$ & $\cdots$ & $\cdots$ & $\ldots$ & $\ldots$ & $\cdots$ & $\cdots$ & $\cdots$ \\
\hline$-0.4,-0.3$ & 778.1 & 292.1 & 33.69 & $\cdots$ & $\cdots$ & $\cdots$ & $\cdots$ & $\cdots$ & $\cdots$ & $\cdots$ & $\cdots$ & $\cdots$ & $\cdots$ & $\cdots$ & $\cdots$ & $\cdots$ & $\cdots$ & $\cdots$ \\
\hline$-0.5,-0.4$ & 692.3 & 202.2 & 17.42 & $\cdots$ & $\cdots$ & $\cdots$ & $\cdots$ & $\cdots$ & $\cdots$ & $\cdots$ & $\cdots$ & $\cdots$ & $\cdots$ & $\cdots$ & $\cdots$ & $\cdots$ & $\cdots$ & $\cdots$ \\
\hline$-0.6,-0.5$ & 600.2 & 135.2 & 3.624 & $\cdots$ & $\cdots$ & $\cdots$ & $\cdots$ & $\cdots$ & $\cdots$ & $\cdots$ & $\cdots$ & $\cdots$ & $\cdots$ & $\cdots$ & $\cdots$ & $\cdots$ & $\cdots$ & $\cdots$ \\
\hline$-0.7,-0.6$ & 497.6 & 85.80 & 0.164 & $\cdots$ & $\cdots$ & $\cdots$ & $\cdots$ & $\cdots$ & $\cdots$ & $\cdots$ & $\cdots$ & $\cdots$ & $\cdots$ & $\cdots$ & $\cdots$ & $\cdots$ & $\cdots$ & $\cdots$ \\
\hline$-0.8,-0.7$ & 418.3 & 44.84 & $\cdots$ & $\cdots$ & $\cdots$ & $\cdots$ & $\cdots$ & $\cdots$ & $\cdots$ & $\cdots$ & $\cdots$ & $\cdots$ & $\cdots$ & $\cdots$ & $\cdots$ & $\cdots$ & $\cdots$ & $\cdots$ \\
\hline$-0.9,-0.8$ & 348.7 & 25.82 & $\cdots$ & $\cdots$ & $\cdots$ & $\cdots$ & $\cdots$ & $\cdots$ & $\cdots$ & $\cdots$ & $\cdots$ & $\cdots$ & $\cdots$ & $\cdots$ & $\cdots$ & $\cdots$ & $\cdots$ & $\cdots$ \\
\hline$-1.0,-0.9$ & 289.2 & 15.18 & $\cdots$ & $\cdots$ & $\cdots$ & $\cdots$ & $\cdots$ & $\cdots$ & $\cdots$ & $\cdots$ & $\cdots$ & $\cdots$ & $\cdots$ & $\cdots$ & $\cdots$ & $\cdots$ & $\cdots$ & $\cdots$ \\
\hline
\end{tabular}

CCQE signal in MiniBooNE. These events originate from the $\mathrm{CC} 1 \pi^{+}$interaction but contain 1 muon and no pions in the final state. In the main analysis, this background is subtracted to obtain the CCQE observables. In order to facilitate comparisons with models (or other analyses) that consider all CCQE-like events as CCQE signal, the effective double differential cross section for the $\mathrm{CC} 1 \pi^{+}$interaction with intranuclear pion absorption is presented in
Table VIII. These values are determined from the NUANCE-event generator corrected to reproduce the MiniBooNE 3-subevent sample and are calculated using Eq. (3) with $\left(d_{j}-b_{j}\right)$ replaced by $b_{j}^{\prime}$, the number of CCQE-like background events. A CCQE-like cross section may be obtained by adding these numbers (Table VIII) with those from Table VI.

TABLE VII. Shape uncertainty on the MiniBooNE $\nu_{\mu}$ CCQE flux-integrated double differential cross section in units of $10^{-42} \mathrm{~cm}^{2} / \mathrm{GeV}$ corresponding to Table VI. The total normalization error is $10.7 \%$.

\begin{tabular}{|c|c|c|c|c|c|c|c|c|c|c|c|c|c|c|c|c|c|c|}
\hline $\cos \theta_{\mu} T_{\mu}(\mathrm{GeV})$ & $2,0.3$ & $0.3,0.4$ & $0.4,0.5$ & $0.5,0.6$ & $0.6,0.7$ & $0.7,0.8$ & $0.8,0.9$ & $0.9,1.0$ & $1.0,1.1$ & $1.1,1$ & $2,1.3$ & $1.3,1.4$ & $1.4,1.5$ & $1.5,1.6$ & $1.6,1.7$ & $1.7,1.8$ & $1.8,1.9$ & $1.9,2.0$ \\
\hline$+0.9,+1.0$ & 684.3 & 1071 & 1378 & 1664 & 1883 & 2193 & 2558 & 3037 & 390 & 3320 & 3037 & 3110 & 2942 & 2424 & 2586 & 2653 & 3254 & 3838 \\
\hline$+0.8,+0.9$ & 905.0 & 1352 & 1754 & 2009 & 2222 & 2334 & 2711 & 2870 & 2454 & 1880 & 1391 & 1036 & 758.7 & 544.3 & 505.5 & 359.6 & $\ldots$ & $\ldots$ \\
\hline$+0.7,+0.8$ & 1134 & 1557 & 1781 & 1845 & 1769 & 1823 & 1873 & 1464 & 963.8 & 601.6 & 339.6 & 184.1 & 170.1 & 230.6 & $\ldots$ & $\ldots$ & $\ldots$ & $\ldots$ \\
\hline$+0.6,+0.7$ & 1435 & 1455 & 1581 & 1648 & 1791 & 1513 & 1068 & 598.2 & 267.2 & 155.1 & 69.28 & 89.01 & $\cdots$ & $\cdots$ & $\cdots$ & $\cdots$ & $\cdots$ & $\cdots$ \\
\hline$+0.5,+0.6$ & 1380 & 1372 & 1434 & 1370 & 1201 & 870.2 & 432.3 & 162.2 & 71.88 & 49.10 & 54.01 & $\cdots$ & $\cdots$ & $\cdots$ & $\cdots$ & $\cdots$ & $\ldots$ & $\ldots$ \\
\hline$+0.4,+0.5$ & 1477 & 1273 & 1365 & 1369 & 1021 & 475.5 & 161.6 & 55.58 & 16.32 & $\cdots$ & $\ldots$ & $\ldots$ & $\cdots$ & $\ldots$ & $\ldots$ & $\ldots$ & $\ldots$ & $\ldots$ \\
\hline$+0.3,+0.4$ & 1267 & 1154 & 1155 & 965.3 & 574.7 & 149.2 & 53.26 & $\ldots$ & $\ldots$ & $\cdots$ & $\cdots$ & $\cdots$ & $\ldots$ & $\ldots$ & $\ldots$ & $\ldots$ & $\ldots$ & $\ldots$ \\
\hline$+0.2,+0.3$ & 1293 & 1105 & 1041 & 742.5 & 250.6 & 77.66 & 110.3 & $\cdots$ & $\ldots$ & $\cdots$ & $\cdots$ & $\cdots$ & $\cdots$ & $\cdots$ & $\ldots$ & $\cdots$ & $\ldots$ & $\ldots$ \\
\hline$+0.1,+0.2$ & 1351 & 1246 & 1048 & 415.1 & 114.3 & 41.02 & $\ldots$ & $\ldots$ & $\ldots$ & $\cdots$ & $\ldots$ & $\ldots$ & $\ldots$ & $\ldots$ & $\ldots$ & $\ldots$ & $\ldots$ & $\ldots$ \\
\hline $0.0,+0.1$ & 1090 & 1078 & 695.5 & 238.2 & 45.96 & $\cdots$ & $\ldots$ & $\cdots$ & $\cdots$ & $\cdots$ & $\cdots$ & $\cdots$ & $\cdots$ & $\cdots$ & $\cdots$ & $\cdots$ & $\cdots$ & $\cdots$ \\
\hline$-0.1,0.0$ & 980.4 & 783.6 & 515.7 & 114.6 & 20.92 & $\ldots$ & $\cdots$ & $\cdots$ & $\cdots$ & $\cdots$ & $\cdots$ & $\cdots$ & $\cdots$ & $\cdots$ & $\cdots$ & $\cdots$ & $\cdots$ & $\cdots$ \\
\hline$-0.2,-0.1$ & 917.7 & 746.9 & 337.5 & 50.92 & 3.422 & $\cdots$ & $\cdots$ & $\cdots$ & $\cdots$ & $\cdots$ & $\cdots$ & $\cdots$ & $\cdots$ & $\cdots$ & $\cdots$ & $\cdots$ & $\cdots$ & $\cdots$ \\
\hline$-0.3,-0.2$ & 922.7 & 586.4 & 215.6 & 55.88 & $\cdots$ & $\ldots$ & $\ldots$ & $\cdots$ & $\cdots$ & $\cdots$ & $\cdots$ & $\cdots$ & $\cdots$ & $\cdots$ & $\cdots$ & $\cdots$ & $\cdots$ & $\cdots$ \\
\hline$-0.4,-0.3$ & 698.0 & 553.3 & 135.3 & $\cdots$ & $\cdots$ & $\ldots$ & $\ldots$ & $\cdots$ & $\cdots$ & $\ldots$ & $\cdots$ & $\cdots$ & $\cdots$ & $\cdots$ & $\cdots$ & $\cdots$ & $\cdots$ & $\cdots$ \\
\hline$-0.5,-0.4$ & 596.9 & 482.6 & 57.73 & $\cdots$ & $\ldots$ & $\ldots$ & $\ldots$ & $\ldots$ & $\ldots$ & $\ldots$ & $\ldots$ & $\cdots$ & $\ldots$ & $\ldots$ & $\ldots$ & $\ldots$ & $\ldots$ & $\ldots$ \\
\hline$-0.6,-0.5$ & 520.8 & 360.7 & 34.63 & $\cdots$ & $\cdots$ & $\cdots$ & $\ldots$ & $\ldots$ & $\cdots$ & $\cdots$ & $\cdots$ & $\cdots$ & $\cdots$ & $\cdots$ & $\cdots$ & $\cdots$ & $\cdots$ & $\cdots$ \\
\hline$-0.7,-0.6$ & 450.2 & 236.6 & 31.22 & $\cdots$ & $\cdots$ & $\cdots$ & $\cdots$ & $\cdots$ & $\cdots$ & $\cdots$ & $\cdots$ & $\cdots$ & $\cdots$ & $\cdots$ & $\cdots$ & $\cdots$ & $\cdots$ & $\cdots$ \\
\hline$-0.8,-0.7$ & 408.8 & 184.4 & $\cdots$ & $\cdots$ & $\cdots$ & $\cdots$ & $\cdots$ & $\cdots$ & $\cdots$ & $\cdots$ & $\cdots$ & $\cdots$ & $\cdots$ & $\cdots$ & $\cdots$ & $\cdots$ & $\cdots$ & $\cdots$ \\
\hline$-0.9,-0.8$ & 339.7 & 107.6 & $\cdots$ & $\cdots$ & $\cdots$ & $\cdots$ & $\cdots$ & $\ldots$ & $\cdots$ & $\ldots$ & $\cdots$ & $\cdots$ & $\cdots$ & $\cdots$ & $\cdots$ & $\cdots$ & $\cdots$ & $\cdots$ \\
\hline$-1.0,-0.9$ & 349.8 & 63.32 & $\ldots$ & $\cdots$ & $\ldots$ & $\ldots$ & $\ldots$ & $\ldots$ & $\ldots$ & $\ldots$ & $\ldots$ & $\ldots$ & $\ldots$ & $\ldots$ & $\cdots$ & $\ldots$ & $\ldots$ & $\ldots$ \\
\hline
\end{tabular}


TABLE VIII. The predicted $\nu_{\mu}$ CCQE-like background flux-integrated double differential cross section in units of $10^{-41} \mathrm{~cm}^{2} / \mathrm{GeV}^{-}$ corresponding to Table VI.

\begin{tabular}{|c|c|c|c|c|c|c|c|c|c|c|c|c|c|c|c|c|c|c|}
\hline $\cos \theta_{\mu} T_{\mu}(\mathrm{GeV})$ & $.2,0.3$ & $0.3,0$ & $0.4,0$ & $0.5,0$ & $0.6,0.7$ & $0.7,0$ & $0.8,0$ & $0.9,1.0$ & 1.0 & 1.1 & $1.2,1$ & $1.3,1.4$ & 1.4 & $1.5,1.6$ & $1.6,1.7$ & $1.7,1.8$ & $1.8,1.9$ & $1.9,2.0$ \\
\hline$+0.9,+1.0$ & 83.6 & 199.8 & 285.3 & 364.2 & 391.1 & 403.7 & 384.3 & 349.2 & 301.4 & 232.7 & 179.2 & 136.1 & 102.0 & 90.73 & 76.55 & 52.36 & 41.47 & 54.50 \\
\hline$+0.8,+0.9$ & 111.6 & 257.4 & 351.0 & 364.3 & 353.2 & 288.9 & 233.8 & 169.5 & 106.6 & 59.81 & 31.21 & 20.89 & 10.10 & 6.008 & 2.376 & 2.859 & $\ldots$ & $\ldots$ \\
\hline$+0.7,+0.8$ & 118.4 & 270.4 & 312.6 & 280.3 & 211.7 & 135.7 & 81.47 & 40.97 & 21.56 & 9.247 & 3.284 & 0.875 & 0.057 & $\ldots$ & $\ldots$ & $\ldots$ & $\ldots$ & $\ldots$ \\
\hline$+0.6,+0.7$ & 118.9 & 260.0 & 252.8 & 183.4 & 101.8 & 52.52 & 19.75 & 7.978 & 2.716 & 0.281 & $\ldots$ & $\ldots$ & $\ldots$ & $\ldots$ & $\ldots$ & $\ldots$ & $\ldots$ & $\ldots$ \\
\hline$+0.5,+0.6$ & 109.0 & 215.2 & 181.4 & 104.6 & 41.87 & 16.33 & 3.643 & 0.492 & 0.004 & $\ldots$ & $\ldots$ & $\ldots$ & $\ldots$ & $\ldots$ & $\ldots$ & $\ldots$ & $\ldots$ & $\ldots$ \\
\hline$+0.4,+0.5$ & 109.2 & 182.0 & 122.4 & 51.26 & 19.76 & 4.193 & 0.183 & $\ldots$ & $\cdots$ & $\ldots$ & $\cdots$ & $\cdots$ & $\ldots$ & $\ldots$ & $\ldots$ & $\ldots$ & $\ldots$ & $\ldots$ \\
\hline$+0.3,+0.4$ & 104.0 & 140.2 & 73.71 & 24.54 & 4.613 & 0.151 & 0.002 & $\ldots$ & $\ldots$ & $\ldots$ & $\cdots$ & $\cdots$ & $\ldots$ & $\ldots$ & $\ldots$ & $\ldots$ & $\ldots$ & $\ldots$ \\
\hline$+0.2,+0.3$ & 93.84 & 107.6 & 48.56 & 10.78 & 0.812 & $\ldots$ & $\ldots$ & $\ldots$ & $\ldots$ & $\ldots$ & $\ldots$ & $\ldots$ & $\ldots$ & $\ldots$ & $\ldots$ & $\ldots$ & $\ldots$ & $\ldots$ \\
\hline$+0.1,+0.2$ & 76.55 & 80.94 & 29.02 & 3.049 & 0.030 & $\cdots$ & $\cdots$ & $\cdots$ & $\cdots$ & $\cdots$ & $\cdots$ & $\cdots$ & $\cdots$ & $\cdots$ & $\cdots$ & $\cdots$ & $\cdots$ & $\cdots$ \\
\hline $0.0,+0.1$ & 67.81 & 52.89 & 13.71 & 0.392 & $\ldots$ & $\ldots$ & $\ldots$ & $\ldots$ & $\ldots$ & $\ldots$ & $\ldots$ & $\ldots$ & $\ldots$ & $\ldots$ & $\ldots$ & $\ldots$ & $\ldots$ & $\ldots$ \\
\hline$-0.1,0.0$ & 58.91 & 37.46 & 5.565 & 0.011 & $\ldots$ & $\ldots$ & $\ldots$ & $\ldots$ & $\ldots$ & $\ldots$ & $\ldots$ & $\ldots$ & $\ldots$ & $\ldots$ & $\ldots$ & $\ldots$ & $\ldots$ & $\ldots$ \\
\hline$-0.2,-0.1$ & 50.47 & 22.49 & 1.048 & $\ldots$ & $\cdots$ & $\ldots$ & $\ldots$ & $\ldots$ & $\ldots$ & $\ldots$ & $\ldots$ & $\ldots$ & $\ldots$ & $\ldots$ & $\ldots$ & $\ldots$ & $\ldots$ & $\ldots$ \\
\hline$-0.3,-0.2$ & 39.03 & 12.58 & 0.118 & $\cdots$ & $\cdots$ & $\ldots$ & $\ldots$ & $\cdots$ & $\cdots$ & $\cdots$ & $\cdots$ & $\cdots$ & $\ldots$ & $\cdots$ & $\ldots$ & $\cdots$ & $\cdots$ & $\cdots$ \\
\hline$-0.4,-0.3$ & 32.41 & 7.575 & 0.061 & $\ldots$ & $\ldots$ & $\ldots$ & $\ldots$ & $\ldots$ & $\ldots$ & $\ldots$ & $\ldots$ & $\ldots$ & $\ldots$ & $\ldots$ & $\ldots$ & $\ldots$ & $\ldots$ & $\ldots$ \\
\hline$-0.5,-0.4$ & 25.72 & 2.529 & 0.080 & $\cdots$ & $\cdots$ & $\cdots$ & $\cdots$ & $\cdots$ & $\cdots$ & $\cdots$ & $\cdots$ & $\cdots$ & $\cdots$ & $\cdots$ & $\cdots$ & $\cdots$ & $\cdots$ & $\cdots$ \\
\hline$-0.6,-0.5$ & 16.78 & 1.063 & 0.009 & $\ldots$ & $\ldots$ & $\ldots$ & $\ldots$ & $\ldots$ & $\ldots$ & $\ldots$ & $\ldots$ & $\ldots$ & $\ldots$ & $\ldots$ & $\ldots$ & $\ldots$ & $\ldots$ & $\ldots$ \\
\hline$-0.7,-0.6$ & 9.963 & 0.280 & 0.002 & $\ldots$ & $\cdots$ & $\ldots$ & $\ldots$ & $\ldots$ & $\ldots$ & $\ldots$ & $\ldots$ & $\ldots$ & $\ldots$ & $\ldots$ & $\ldots$ & $\ldots$ & $\ldots$ & $\ldots$ \\
\hline$-0.8,-0.7$ & 5.005 & 0.244 & $\ldots$ & $\ldots$ & $\ldots$ & $\ldots$ & $\ldots$ & $\ldots$ & $\ldots$ & $\ldots$ & $\ldots$ & $\ldots$ & $\ldots$ & $\ldots$ & $\ldots$ & $\ldots$ & $\ldots$ & $\ldots$ \\
\hline$-0.9,-0.8$ & 4.877 & 0.067 & $\cdots$ & $\cdots$ & $\cdots$ & $\cdots$ & $\cdots$ & $\cdots$ & $\cdots$ & $\cdots$ & $\cdots$ & $\cdots$ & $\cdots$ & $\cdots$ & $\cdots$ & $\cdots$ & $\cdots$ & $\cdots$ \\
\hline$-1.0,-0.9$ & 3.092 & 0.013 & $\ldots$ & $\cdots$ & $\cdots$ & $\ldots$ & $\ldots$ & $\cdots$ & $\ldots$ & $\cdots$ & $\cdots$ & $\cdots$ & $\ldots$ & $\ldots$ & $\ldots$ & $\ldots$ & $\ldots$ & $\ldots$ \\
\hline
\end{tabular}

\section{CCQE flux-integrated single differential cross section}

Table IX contains the flux-integrated CCQE single differential cross section $\left(\frac{d \sigma}{d Q_{\mathrm{QE}}^{2}}\right)$ in bins of $Q_{\mathrm{QE}}^{2} \cdot Q_{\mathrm{QE}}^{2}$ is as defined in Eq. (2). The shape error and CCQE-like background prediction is also reported. The corresponding plot is shown in Fig. 14.

\section{Flux unfolded CCQE cross section}

Table X contains the flux-unfolded $\nu_{\mu}$ CCQE cross section values $\sigma\left[E_{\nu}^{\mathrm{QE}, \mathrm{RFG}}\right]$ in bins of $E_{\nu}^{\mathrm{QE}, \mathrm{RFG}} \cdot E_{\nu}^{\mathrm{QE}, \mathrm{RFG}}$ is as defined in Eq. (1). The shape and total errors along with the CCQE-like background are also reported. The corresponding plot is shown in Fig. 15.

TABLE IX. The MiniBooNE $\nu_{\mu}$ CCQE flux-integrated single differential cross section, errors, and predicted CCQE-like background in bins of $Q_{\mathrm{QE}}^{2}$. The total normalization error is $10.7 \%$.

\begin{tabular}{lccc}
\hline \hline$Q_{\mathrm{QE}}^{2}\left(\mathrm{GeV}^{2}\right)$ & $\frac{d \sigma}{d Q_{\mathrm{QE}}^{2}}\left(\mathrm{~cm}^{2} / \mathrm{GeV}^{2}\right)$ & shape error $\left(\mathrm{cm}^{2} / \mathrm{GeV}^{2}\right)$ & $\mathrm{CCQE}-\mathrm{like} \mathrm{bkgd}\left(\mathrm{cm}^{2} / \mathrm{GeV}^{2}\right)$ \\
\hline $0.00-0.05$ & $7.681 \times 10^{-39}$ & $1.493 \times 10^{-39}$ & $3.876 \times 10^{-39}$ \\
$0.05-0.10$ & $1.457 \times 10^{-38}$ & $1.180 \times 10^{-39}$ & $3.961 \times 10^{-39}$ \\
$0.10-0.15$ & $1.684 \times 10^{-38}$ & $9.720 \times 10^{-40}$ & $3.671 \times 10^{-39}$ \\
$0.15-0.20$ & $1.703 \times 10^{-38}$ & $8.216 \times 10^{-40}$ & $3.064 \times 10^{-39}$ \\
$0.20-0.25$ & $1.589 \times 10^{-38}$ & $5.134 \times 10^{-40}$ & $2.522 \times 10^{-39}$ \\
$0.25-0.30$ & $1.449 \times 10^{-38}$ & $3.983 \times 10^{-40}$ & $2.040 \times 10^{-39}$ \\
$0.30-0.35$ & $1.329 \times 10^{-38}$ & $3.386 \times 10^{-40}$ & $1.633 \times 10^{-39}$ \\
$0.35-0.40$ & $1.172 \times 10^{-38}$ & $2.629 \times 10^{-40}$ & $1.290 \times 10^{-39}$ \\
$0.40-0.45$ & $1.030 \times 10^{-38}$ & $2.457 \times 10^{-40}$ & $1.018 \times 10^{-39}$ \\
$0.45-0.50$ & $8.852 \times 10^{-39}$ & $2.975 \times 10^{-40}$ & $7.874 \times 10^{-40}$ \\
$0.50-0.60$ & $7.164 \times 10^{-39}$ & $3.193 \times 10^{-40}$ & $5.524 \times 10^{-40}$ \\
$0.60-0.70$ & $5.425 \times 10^{-39}$ & $3.212 \times 10^{-40}$ & $3.532 \times 10^{-40}$ \\
$0.70-0.80$ & $4.032 \times 10^{-39}$ & $3.442 \times 10^{-40}$ & $2.302 \times 10^{-40}$ \\
$0.80-1.00$ & $2.713 \times 10^{-39}$ & $2.885 \times 10^{-40}$ & $1.339 \times 10^{-40}$ \\
$1.00-1.20$ & $1.620 \times 10^{-39}$ & $2.250 \times 10^{-40}$ & $6.398 \times 10^{-41}$ \\
$1.20-1.50$ & $9.915 \times 10^{-40}$ & $1.407 \times 10^{-40}$ & $2.466 \times 10^{-41}$ \\
$1.50-2.00$ & $5.474 \times 10^{-40}$ & $2.504 \times 10^{-41}$ & $3.645 \times 10^{-42}$ \\
\hline \hline
\end{tabular}


TABLE X. The MiniBooNE $\nu_{\mu}$ CCQE flux-unfolded cross section, errors, and predicted CCQE-like background in bins of $E_{\nu}^{\mathrm{QEE}, \mathrm{RFG}}$.

\begin{tabular}{lcccc}
\hline \hline$E_{\nu}^{\text {QE,RFG }}(\mathrm{GeV})$ & $\sigma\left(\mathrm{cm}^{2}\right)$ & shape error $\left(\mathrm{cm}^{2}\right)$ & total error $\left(\mathrm{cm}^{2}\right)$ & CCQE-like bkgd $\left(\mathrm{cm}^{2}\right)$ \\
\hline $0.40-0.45$ & $7.985 \times 10^{-39}$ & $1.997 \times 10^{-39}$ & $1.997 \times 10^{-39}$ & $1.731 \times 10^{-39}$ \\
$0.45-0.50$ & $8.261 \times 10^{-39}$ & $1.455 \times 10^{-39}$ & $1.532 \times 10^{-39}$ & $1.865 \times 10^{-39}$ \\
$0.50-0.55$ & $8.809 \times 10^{-39}$ & $1.169 \times 10^{-39}$ & $1.330 \times 10^{-39}$ & $1.951 \times 10^{-39}$ \\
$0.55-0.60$ & $9.530 \times 10^{-39}$ & $9.537 \times 10^{-40}$ & $1.209 \times 10^{-39}$ & $1.978 \times 10^{-39}$ \\
$0.60-0.65$ & $1.013 \times 10^{-38}$ & $7.575 \times 10^{-40}$ & $1.124 \times 10^{-39}$ & $1.941 \times 10^{-39}$ \\
$0.65-0.70$ & $1.071 \times 10^{-38}$ & $6.000 \times 10^{-40}$ & $1.089 \times 10^{-39}$ & $1.878 \times 10^{-39}$ \\
$0.70-0.75$ & $1.111 \times 10^{-38}$ & $4.496 \times 10^{-40}$ & $1.065 \times 10^{-39}$ & $1.770 \times 10^{-39}$ \\
$0.75-0.80$ & $1.155 \times 10^{-38}$ & $3.151 \times 10^{-40}$ & $1.078 \times 10^{-39}$ & $1.672 \times 10^{-39}$ \\
$0.80-0.90$ & $1.202 \times 10^{-38}$ & $1.954 \times 10^{-40}$ & $1.129 \times 10^{-39}$ & $1.528 \times 10^{-39}$ \\
$0.90-1.00$ & $1.230 \times 10^{-38}$ & $2.714 \times 10^{-40}$ & $1.217 \times 10^{-39}$ & $1.334 \times 10^{-39}$ \\
$1.00-1.10$ & $1.258 \times 10^{-38}$ & $4.952 \times 10^{-40}$ & $1.359 \times 10^{-39}$ & $1.187 \times 10^{-39}$ \\
$1.10-1.30$ & $1.258 \times 10^{-38}$ & $9.122 \times 10^{-40}$ & $1.662 \times 10^{-39}$ & $1.005 \times 10^{-39}$ \\
$1.30-1.50$ & $1.278 \times 10^{-38}$ & $1.417 \times 10^{-39}$ & $2.116 \times 10^{-39}$ & $7.944 \times 10^{-40}$ \\
$1.50-2.00$ & $1.236 \times 10^{-38}$ & $1.991 \times 10^{-39}$ & $2.613 \times 10^{-39}$ & $4.822 \times 10^{-40}$ \\
\hline \hline
\end{tabular}

[1] A. A. Aguilar-Arevalo et al. (The MiniBooNE Collaboration), Phys. Rev. Lett. 98, 231801 (2007).

[2] A. A. Aguilar-Arevalo et al. (The MiniBooNE Collaboration), Phys. Rev. Lett. 102, 101802 (2009).

[3] A. A. Aguilar-Arevalo et al. (The MiniBooNE Collaboration), Phys. Rev. Lett. 103, 061802 (2009).

[4] A. A. Aguilar-Arevalo et al. (The MiniBooNE Collaboration), Phys. Rev. Lett. 103, 111801 (2009).

[5] D.S. Ayres et al. (NOvA Collaboration), arXiv:hep-ex/ 0503053 .

[6] Y. Itow et al. (T2K Collaboration), arXiv:hep-ex/0106019.

[7] G. P. Zeller, arXiv:hep-ex/0312061.

[8] R. A. Smith and E. J. Moniz, Nucl. Phys. B43, 605 (1972); B101, 547(E) (1975).

[9] V. Bernard et al., J. Phys. G 28, R1 (2002).

[10] V. Lyubushkin et al. (NOMAD Collaboration), Eur. Phys. J. C 63, 355 (2009).

[11] A. A. Aguilar-Arevalo et al. (MiniBooNE Collaboration), Phys. Rev. Lett. 100, 032301 (2008); Teppei Katori, in The 5th International Workshop on Neutrino-Nucleus Interactions in the Few-GeV Region, edited by Geralyn P. Zeller, Jorge G. Morfin, Flavio Cavanna, AIP Conf. Proc. No. 967 (AIP, New York, 2007), p. 123.

[12] R. Gran et al. (K2K Collaboration), Phys. Rev. D 74, 052002 (2006).

[13] X. Espinal and F. Sanchez (K2K Collaboration), in The 5th International Workshop on Neutrino-Nucleus Interactions in the Few-GeV Region, edited by Geralyn P. Zeller, Jorge G. Morfin, Flavio Cavanna, AIP Conf. Proc. No. 967 (AIP, New York, 2007), p. 117.

[14] J.L. Alcaraz-Aunion and J. Walding (SciBooNE Collaboration), in Sixth International Workshop on Neutrino-Nucleus Interactions in the Few-GeV Region (NUINT-09), edited by Federico Sanchez, M. Sorel, and Luis Alvarez-Ruso, AIP Conf. Proc. No. 1189 (AIP, New York, 2009), p. 145.

[15] M. Dorman (MINOS Collaboration), in Sixth International Workshop on Neutrino-Nucleus Interactions in the Few-GeV Region (NUINT-09), edited by Federico Sanchez, M. Sorel, and Luis Alvarez-Ruso, AIP
Conf. Proc. No. 1189 (AIP, New York, 2009), pp. 133; M. E. Dorman, thesis, FERMILAB, 2008.

[16] J.E. Amaro et al., Phys. Rev. C 71, 015501 (2005); 75, 034613 (2007); A. M. Ankowski et al., Phys. Rev. C 77, 044311 (2008); O. Benhar et al., Phys. Rev. D 72, 053005 (2005); Phys. Rev. D 80, 073003 (2009); A. Butkevich et al., Phys. Rev. C 76, 045502 (2007); 80, 014610 (2009); N. Jachowicz et al., Phys. Rev. C 73, 024607 (2006); T. Leitner et al., Phys. Rev. C 73, 065502 (2006); 79, 034601 (2009); A. Meucci et al., Nucl. Phys. A739, 277 (2004); J. Nieves et al., Phys. Rev. C 73, 025504 (2006); S. K. Singh et al., arXiv:0808.2103.

[17] M. Martini et al., Phys. Rev. C 80, 065501 (2009).

[18] S. Agostinelli et al. (GEANT4 Collaboration), Nucl. Instrum. Methods Phys. Res., Sect. A 506, 250 (2003).

[19] A. A. Aguilar-Arevalo et al. (MiniBooNE Collaboration), Phys. Rev. D 79, 072002 (2009).

[20] M. Catanesi et al. (HARP Collaboration), Eur. Phys. J. C 52, 29 (2007).

[21] J.R. Sanford and C. L. Wang, BNL Report No. 11299 1967.

[22] A. A. Aguilar-Arevalo et al. (MiniBooNE Collaboration), Nucl. Instrum. Methods Phys. Res., Sect. A 599, 28 (2009).

[23] CERN Program Library Long Writeup CERN W5013, 1993.

[24] C. Zeitnitz and T. A. Gabriel, Nucl. Instrum. Methods Phys. Res., Sect. A 349, 106 (1994).

[25] D. Ashery et al., Phys. Rev. C 23, 2173 (1981); M. K. Jones et al., Phys. Rev. C 48, 2800 (1993); R. D. Ransome et al., Phys. Rev. C 45, R509 (1992).

[26] F. Binon et al., Nucl. Phys. B17, 168 (1970).

[27] R. B. Patterson, thesis, FERMILAB 2007.

[28] D. Casper, Nucl. Phys. B, Proc. Suppl. 112, 161 (2002).

[29] D. Rein and L. M. Sehgal, Ann. Phys. (N.Y.) 133, 79 (1981).

[30] D. Rein and L. M. Sehgal, Nucl. Phys. B223, 29 (1983).

[31] M. Gluck, E. Reya, and A. Vogt, Eur. Phys. J. C 5, 461 (1998). 
[32] A. Bodek and U. K. Yang, in Tenth Mexican School on Particles and Fields, edited by Myriam Mondragón, Umberto Cotti, and Gilberto Tavares-Velasco, AIP Conf. Proc. 670 (AIP, New York, 2003), p. 110.

[33] H. Budd, A. Bodek, and J. Arrington, arXiv:hep-ex/ 0308005 .

[34] K. F. Liu et al., Phys. Rev. Lett. 74, 2172 (1995).

[35] E. J. Moniz et al., Phys. Rev. Lett. 26, 445 (1971).

[36] G. Garvey et al., Phys. Rev. C 48, 761 (1993).

[37] R. P. Feynman, M. Kislinger, and F. Ravndal, Phys. Rev. D 3, 2706 (1971).

[38] J. A. Novak, in Sixth International Workshop on NeutrinoNucleus Interactions in the Few-GeV Region (NUINT-09), edited by Federico Sanchez, M. Sorel, and Luis AlvarezRuso, AIP Conf. Proc. No. 1189 (AIP, New York, 2009), pp. 243-248.

[39] M. Hasegawa et al. (K2K Collaboration), Phys. Rev. Lett. 95, 252301 (2005).

[40] A. A. Aguilar-Arevalo et al. (MiniBooNE Collaboration), Phys. Lett. B 664, 41 (2008); Phys. Rev. D 81, 013005 (2010).

[41] K. Hiraide et al. (SciBooNE Collaboration), Phys. Rev. D 78, 112004 (2008).

[42] W. Reuter et al., Phys. Rev. C 26, 806 (1982).

[43] V. Flaminio et al., CERN Report No. HERA-83-01, 1983; CERN Report No. HERA-83-02, 1983; CERN Report No. HERA-84-01, 1984.

[44] D. Casper (private communication).

[45] B. Hahn, D. G. Ravenhall, and R. Hofstadter, Phys. Rev. 101, 1131 (1956).

[46] E. A. Hawker, Nucl. Phys. B, Proc. Suppl. 139, 260 (2005).

[47] T. Suzuki et al., Phys. Rev. C 35, 2212 (1987).
[48] A. A. Aguilar-Arevalo et al. (MiniBooNE Collaboration), Phys. Rev. Lett. 103, 081801 (2009).

[49] M. J. Wilking, thesis, FERMILAB, 2009-27.

[50] R.H. Nelson, in Sixth International Workshop on Neutrino-Nucleus Interactions in the Few-GeV Region (NUINT-09), edited by Federico Sanchez, M. Sorel, and Luis Alvarez-Ruso, AIP Conf. Proc. No. 1189 (AIP, New York, 2009), pp. 201206.

[51] R. B. Patterson et al., Nucl. Instrum. Methods Phys. Res., Sect. A 608, 206 (2009).

[52] J. Grange, in Sixth International Workshop on NeutrinoNucleus Interactions in the Few-GeV Region (NUINT-09), edited by Federico Sanchez, M. Sorel, and Luis AlvarezRuso, AIP Conf. Proc. No. 1189 (AIP, New York, 2009), pp. 331-334.

[53] G. D’Agostini, Nucl. Instrum. Methods Phys. Res., Sect. A 362, 487 (1995).

[54] G. Cowan, Statistical Data Analysis (Oxford Science Publications, New York, 1998).

[55] T. Katori, thesis, FERMILAB, 2008-64.

[56] L. B. Auerbach et al. (LSND Collaboration), Phys. Rev. C 66, 015501 (2002).

[57] A. A. Aguilar-Arevalo et al. (SciBooNE Collaboration), arXiv:hep-ex/0601022.

[58] M. Soderberg (MicroBooNE Collaboration), in Sixth International Workshop on Neutrino-Nucleus Interactions in the Few-GeV Region (NUINT-09), edited by Federico Sanchez, M. Sorel, and Luis Alvarez-Ruso, AIP Conf. Proc. No. 1189 (AIP, New York, 2009), p. 83.

[59] D. Drakoulakos et al. (MINERvA Collaboration), arXiv: hep-ex/0405002.

[60] http://www-boone.fnal.gov/for_physicists/data_release/ ccqe. 